$32044107266 \quad 686$

Tat

487

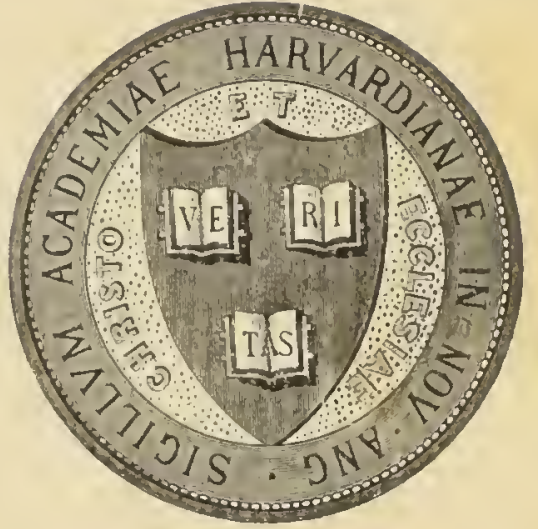

HARVARD UNIVERSITY

I I BR AR Y

OF THE

GRAY HERBARIUM

Received 14 April 1911 




\section{A SYSTEM OE \\ BOTANICAL ANALYSIS \\ APPLIED TO JHE}

I) I A G OSIS OF

BRITISH NATURAL ORDERS.

firor the ellse of a dignmers.

BiS

W. HANDSEL GRTFFTTHS, PH.D.

L O N D O N :

WYMAN \& SONS, 74-5, GREAT QUEEN STRERT, LINCOLN'S-INN IIIELDS.

1870. 



\section{A SYSTLA OF \\ BOTANICAL ANALYSIS \\ - ApPlied TO THE}

DIAGNOSIS OF BRITISH NATURAL ORDERS. 



\title{
1 SYYSTEM OF
}

\section{BOTANICAL ANALYSIS}

APPALI) TO THF

\author{
i) I G GOSIS O F
}

\section{BRITISH NATURAL ORDERS.}

fror the extse of a derinmers.

W. HANDSEL GRIFETTHS, Ph.D.

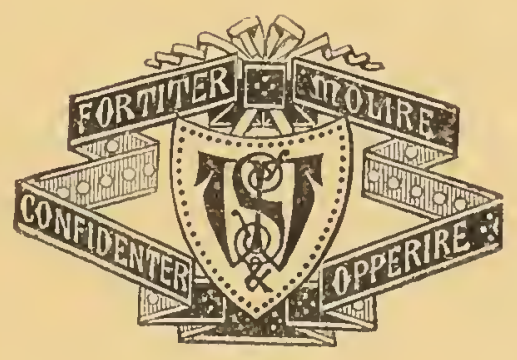

L, O N I) $\mathrm{N}$ :

WYMAN \& SONS, T4-n, GREAT ULGJN STLELT, LINCOLN'S-LNA FIELDS. 
Afm. 14,1911

Gray Herbarium

Harvard University.

LONDON:

WYIAN AND SONA, FHINTER, GRHAT QLUEN STENBT LIN COLN'S-INA YIELUS\& W. C. 


\section{PREFACE.}

I'HE following pages were originally written for, and are now published at the request of, the Author's pupils.

The object which the Compilation is intended to fulfil is to afford to students commencing the study of Botanical Classification a concise, simple, and systematic guide to the Diagnosis of our British Natural Orders.

In the selection of diagnostic data, reference has been had rather to facility of recognition than to morphological significance, and, as the Work is morely designed for practical analysis in the field, almust all malter has been exchuded which is not essential for the pruposes of diagnosis. It should be borne in mind that the characteristics specified as pertaining to Orders are not always without exception, and in sume cases are applicalle culy to British repurscututives. The attention of beginners should also be directed to the fact that in the following System, as in all methods of Artificial Analysis, the Orders are not urranged accorling to thuir Nutural Affinities, in some cases Order's being placed in apposition which should be widely separated, and vice rersû.

Inasinuch as it is presumed that no student will commence the study of Systematic Botany withont laving previously mastered the outlines of Morphology, technicalities have bcen freely used throughout the Work.*

* It is necessary to state that in the following pages the term Polycarpallary signifes an orary composed of more than one carpel; Phurloculer, the cxistence

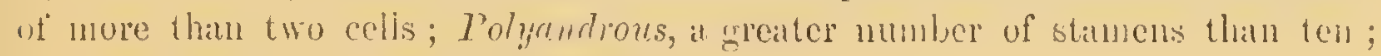
and higundrous, ten or fewer stumens. 
Some of the Orders are printed in itulics, to signify that they do not properly belong to the Group under which they are so printed, but that some of their representatives exhibit approximative characters of the Gromp.

Very few words are needed as to the method of using the following pages. In examining a plant, it is cousecutively referred to its Sub-kingdom, Division, Subdivision, Class, Subclass, and Group; the analysis of the Group is then followed, and the Order of the plant arrived at. When the student has diagnosed the Order, he should refer to his text-book, and study therefrom the cliaracteristics of the Order in extenso. It is hoped that by this means the study of Systematic Botany will be fucilitated for beginners, and rendered a pleasant, as well as a profitable, expenditure of time.

The Author, in conclusion, desires to acknowledge, as valuable sources of information, the works of Bentham, Balfour, Bentley, Henslow, Henfrey, Hooker and Arnott, Lindley, Oliver, and many others; and he wonld record his gratitude to those gentlemen who have so kindly afforded him their assistance and advice.

\section{W. IIANDSEL GRIFFI'THS.}

London, ILuy, 1870. 


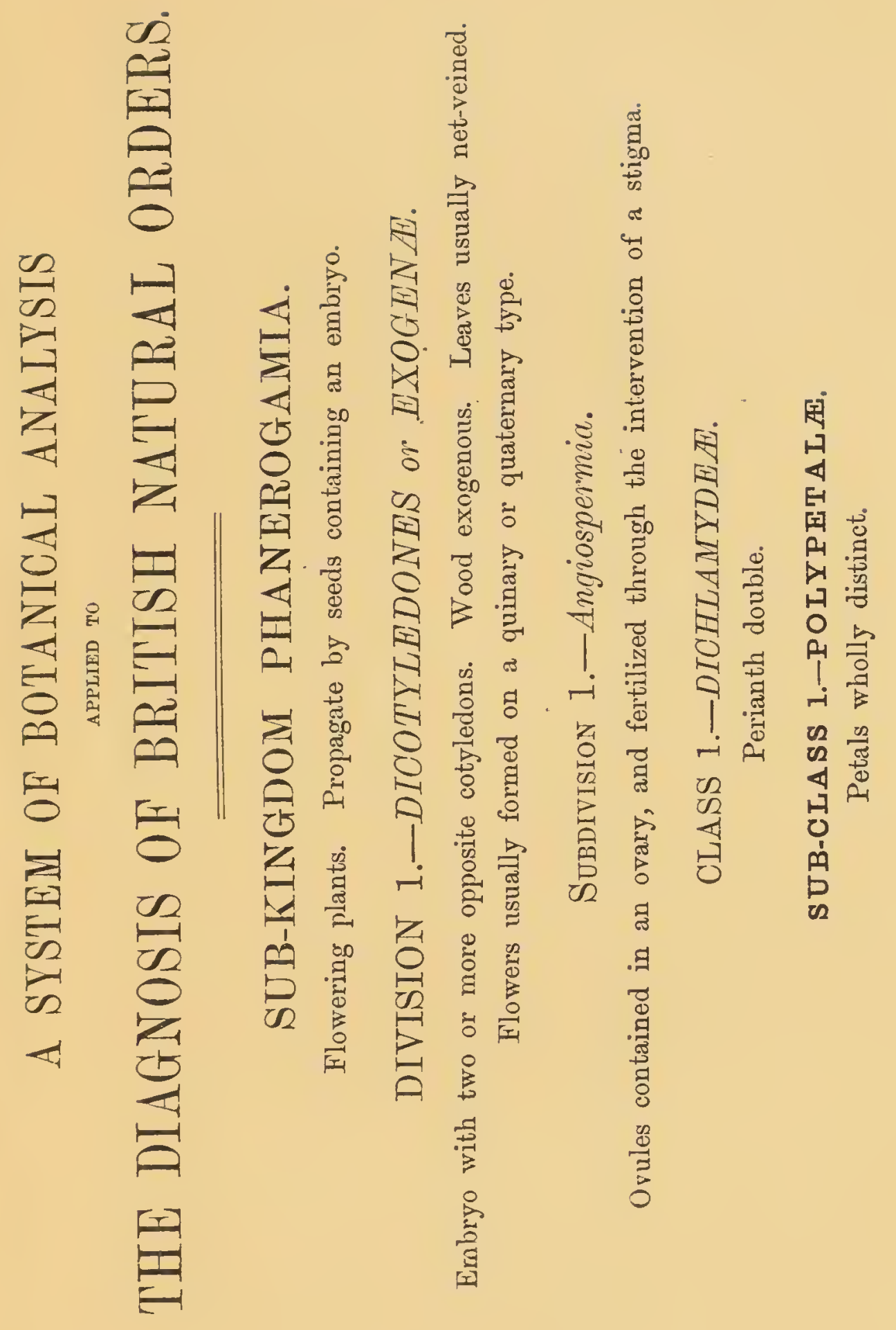




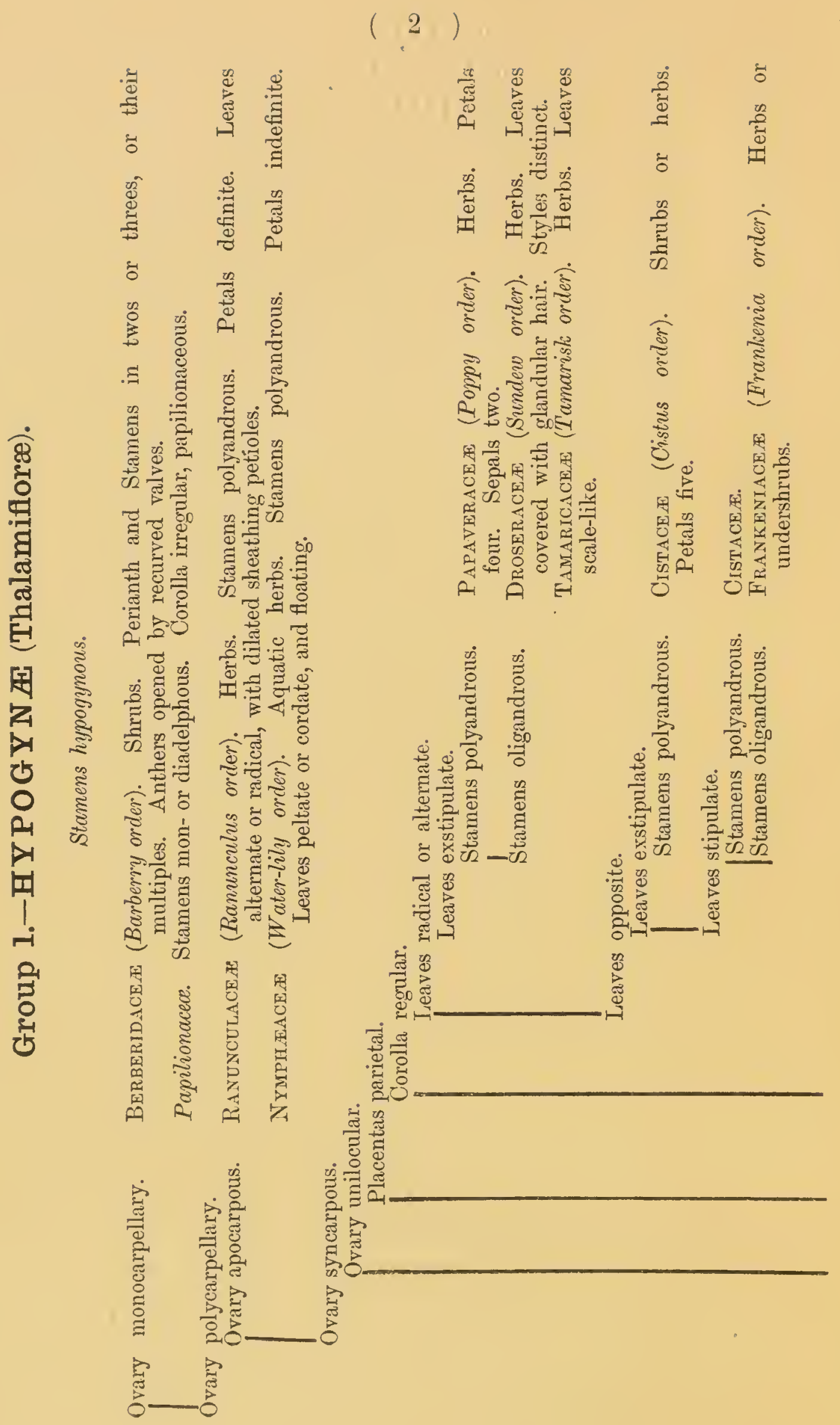




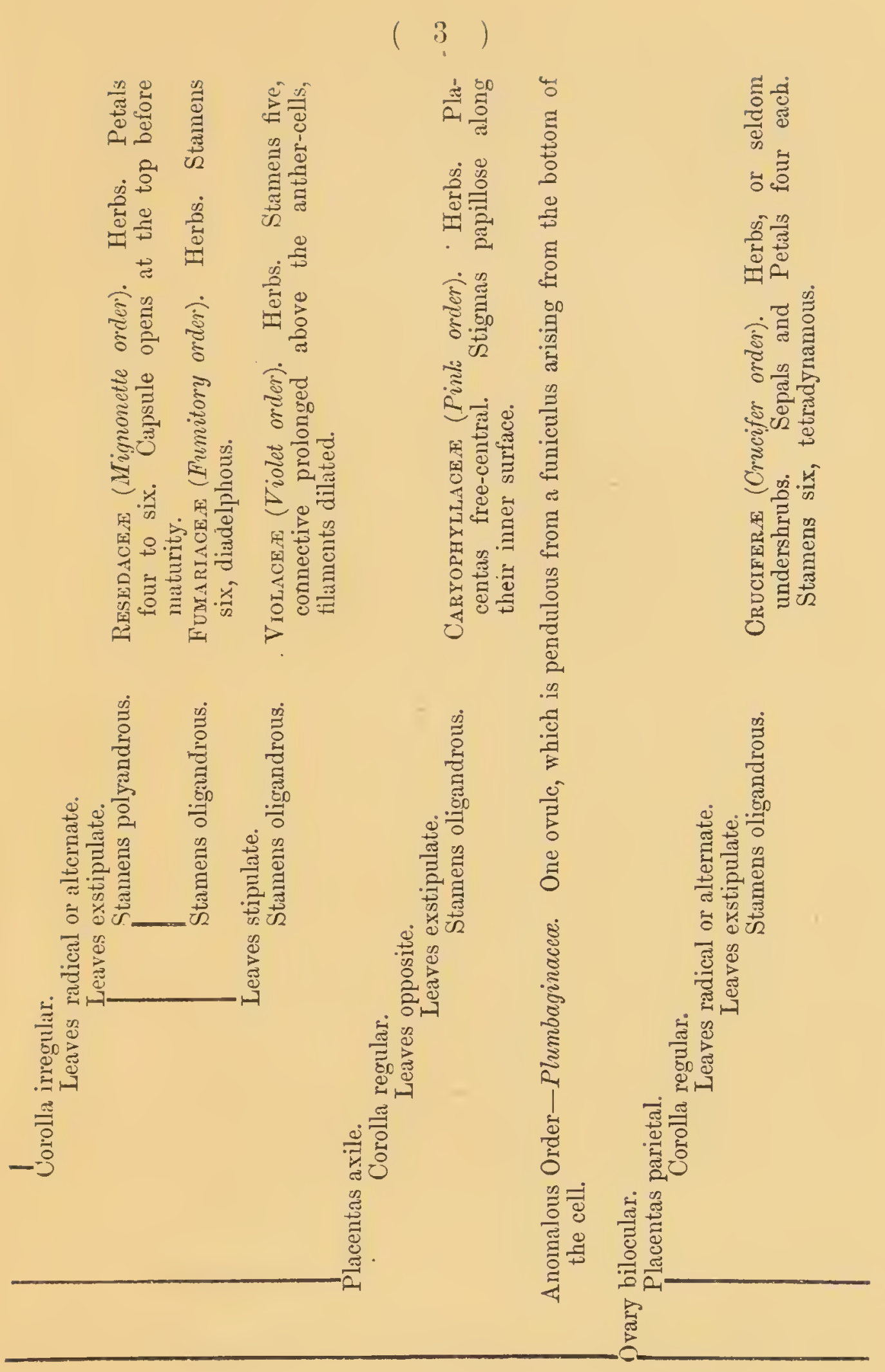


(4.)
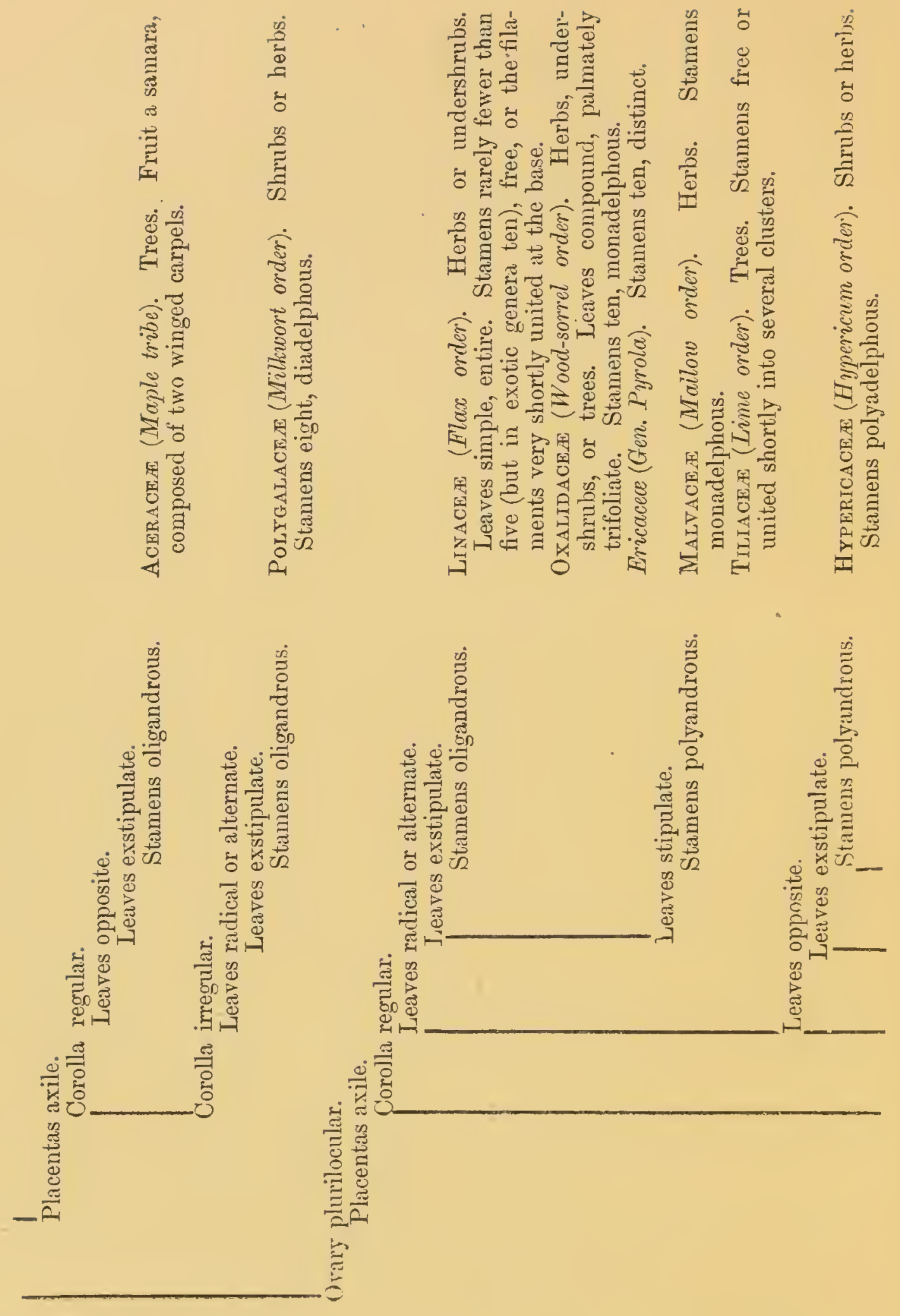

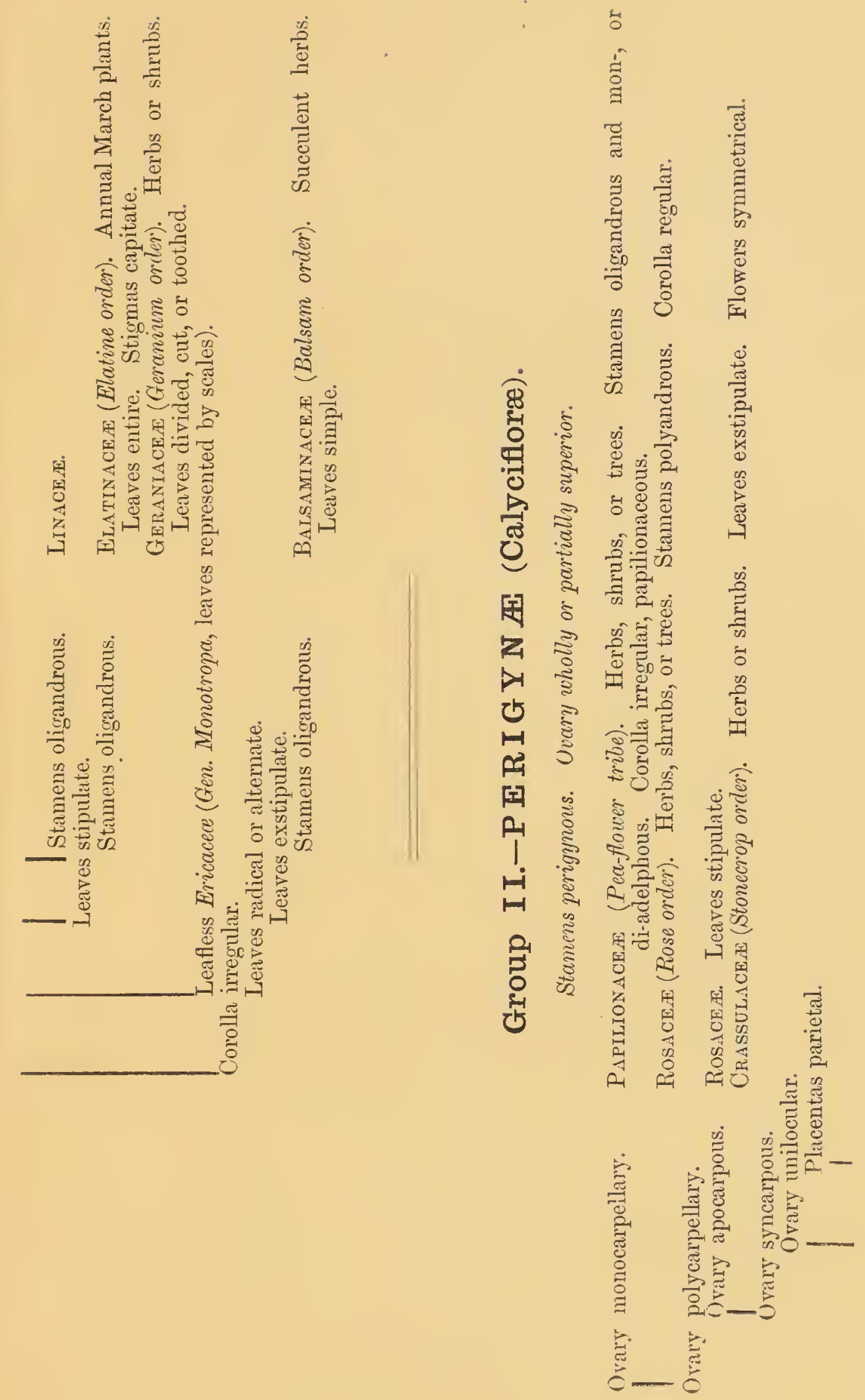

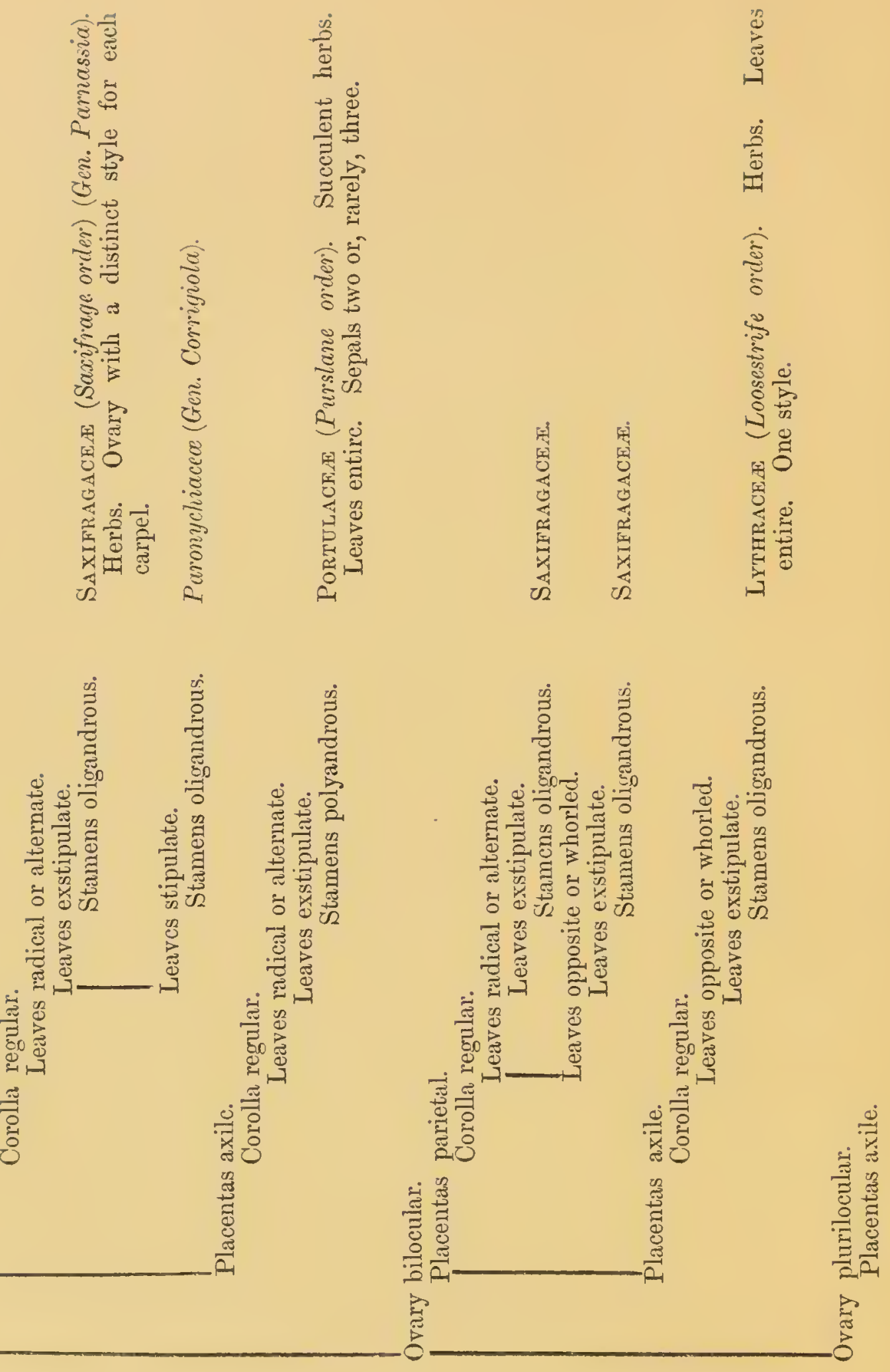


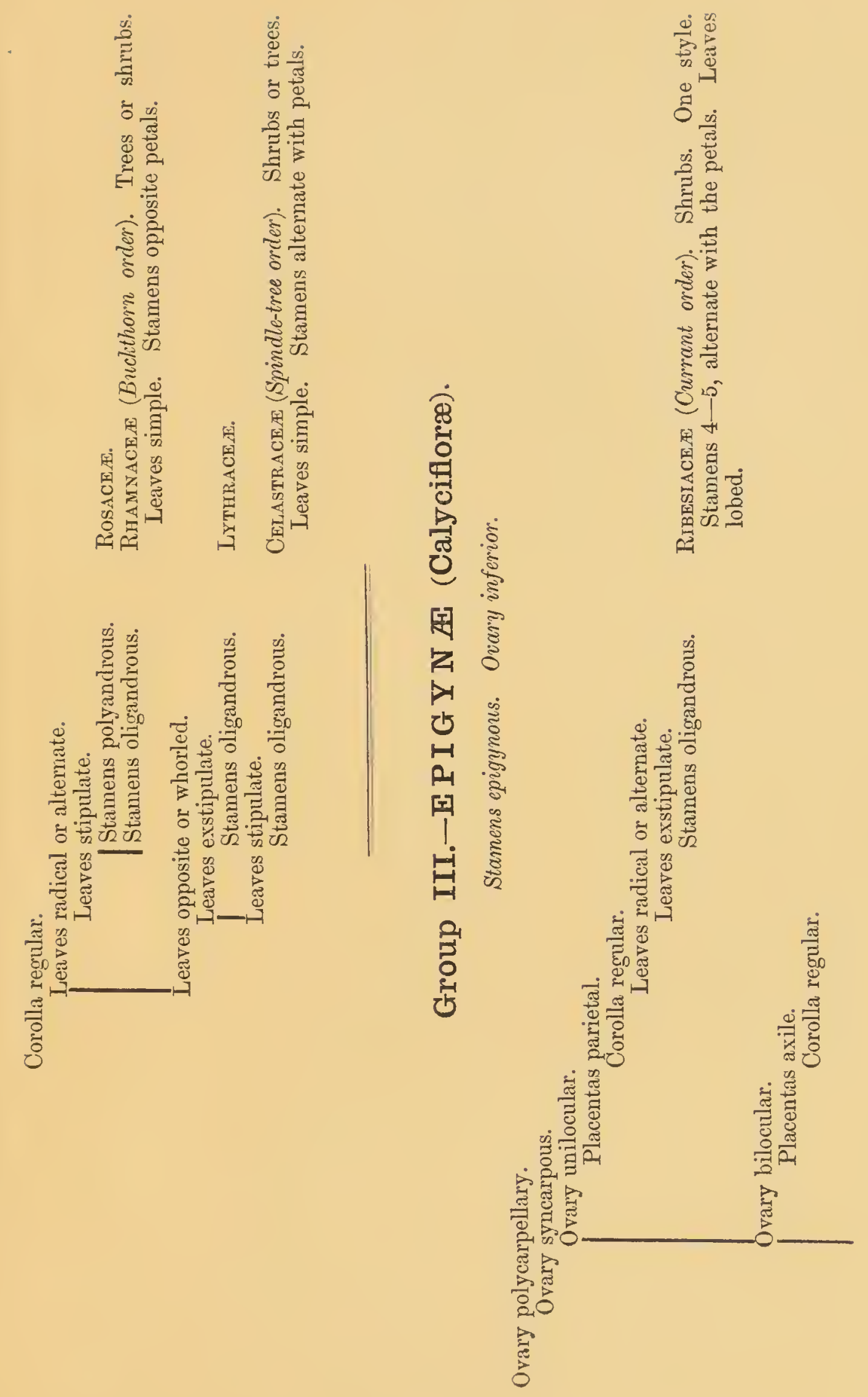




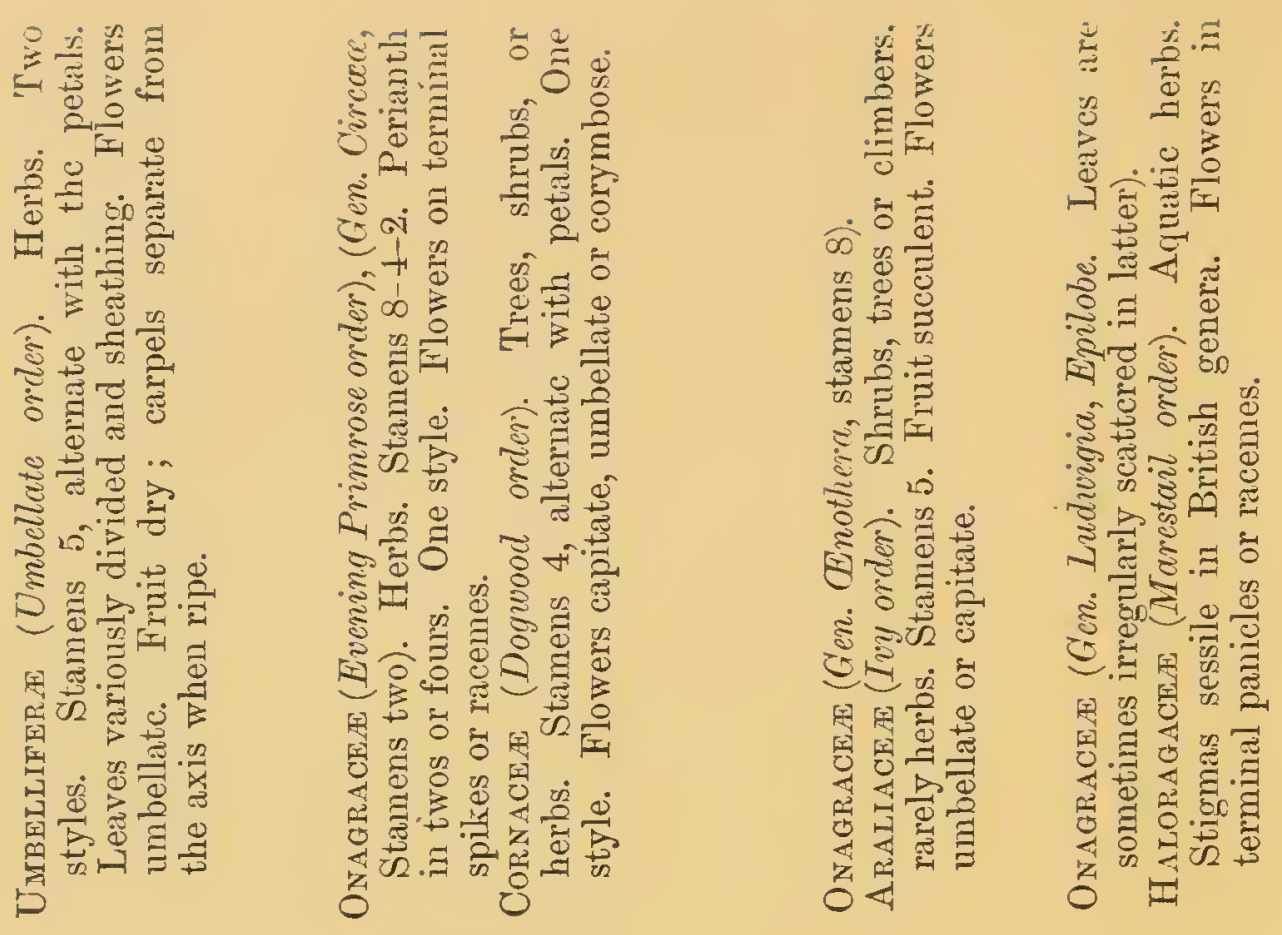
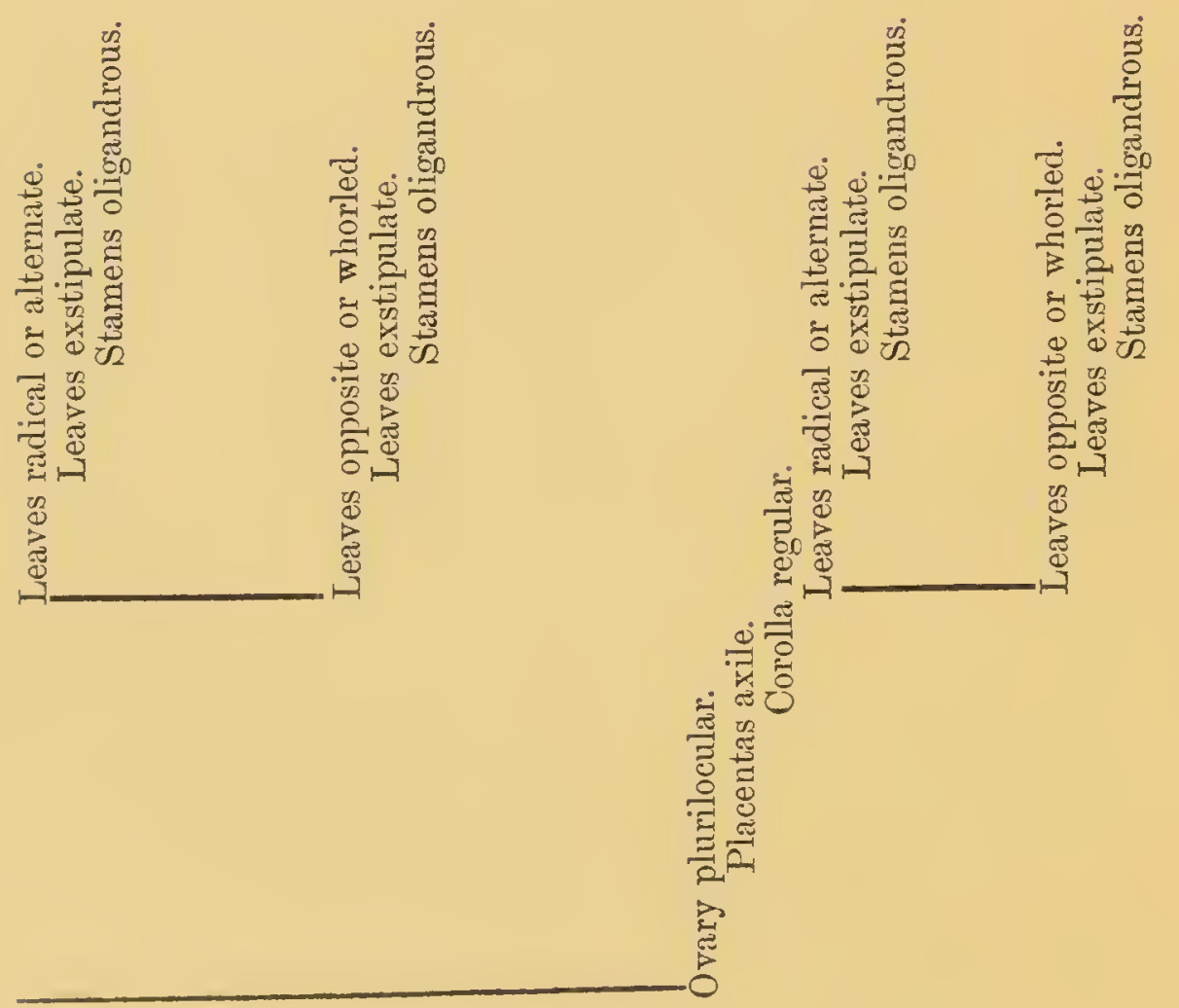


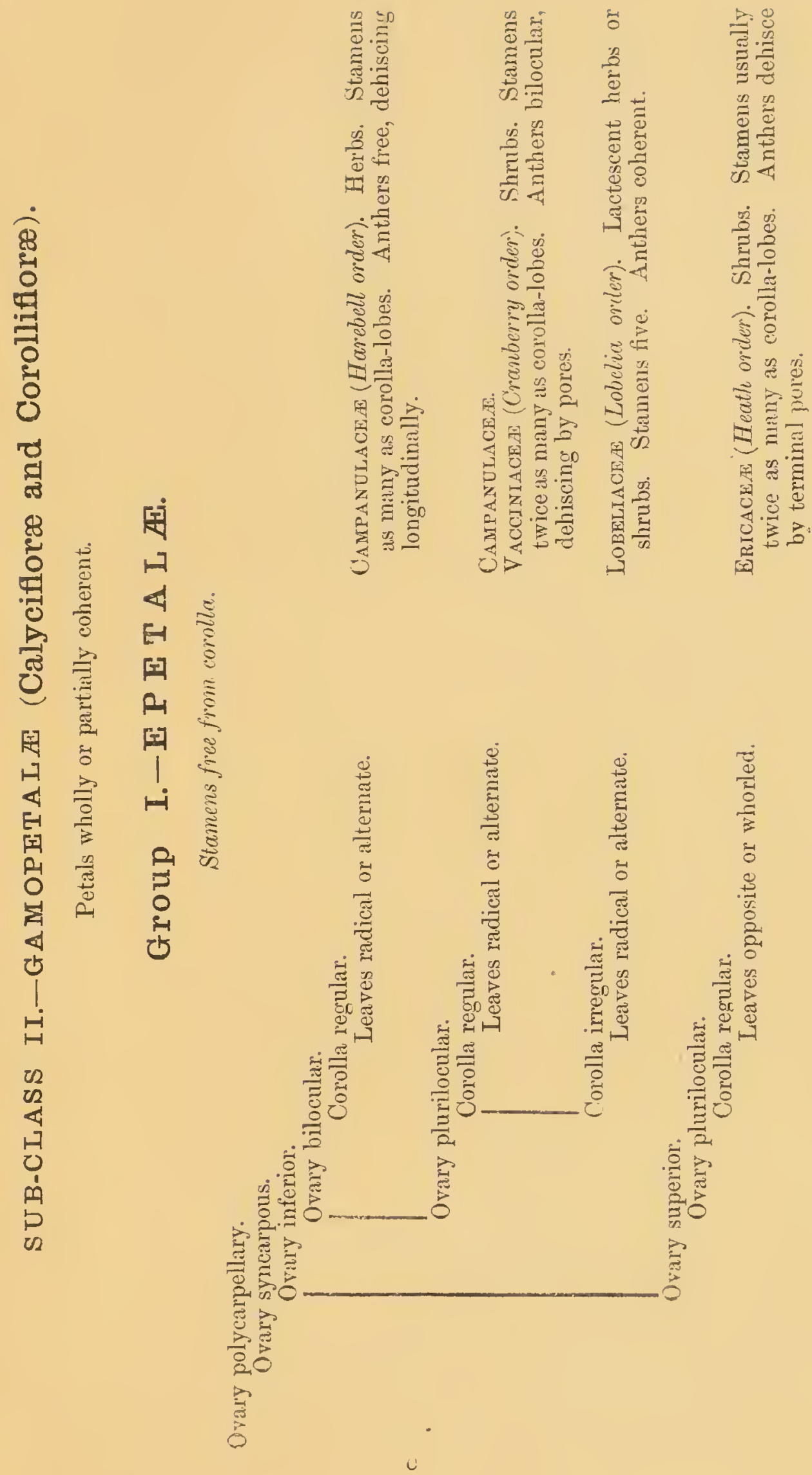




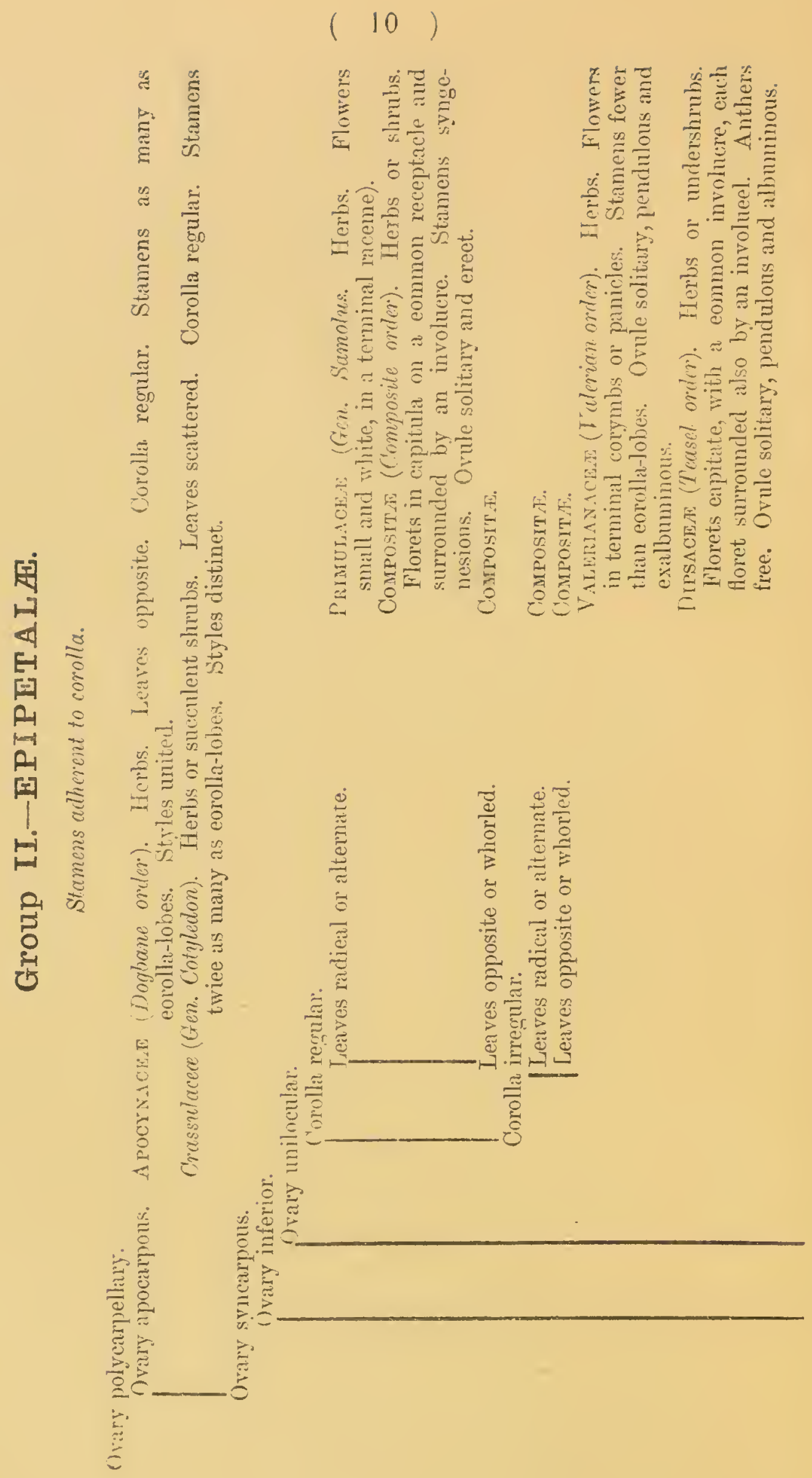



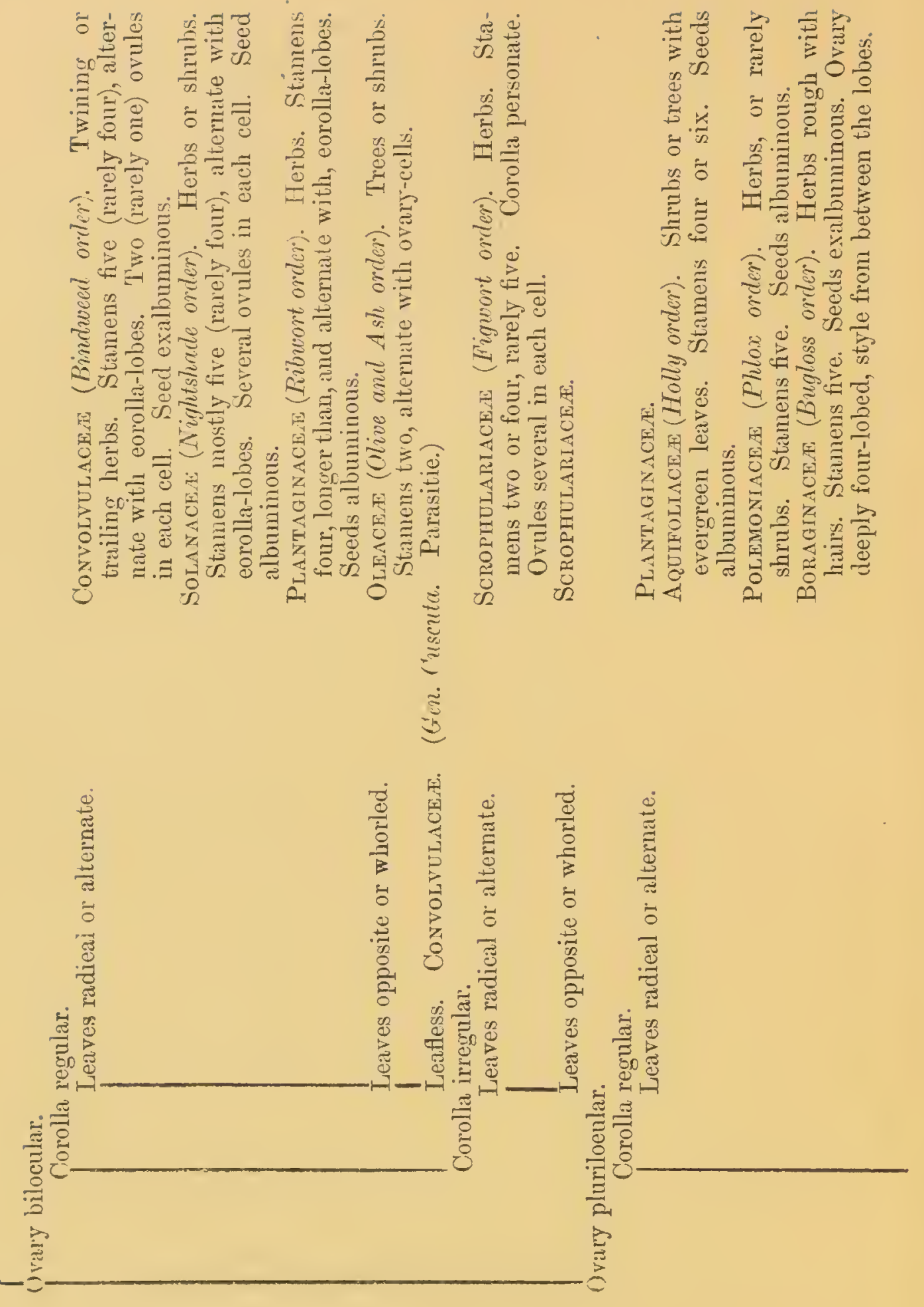

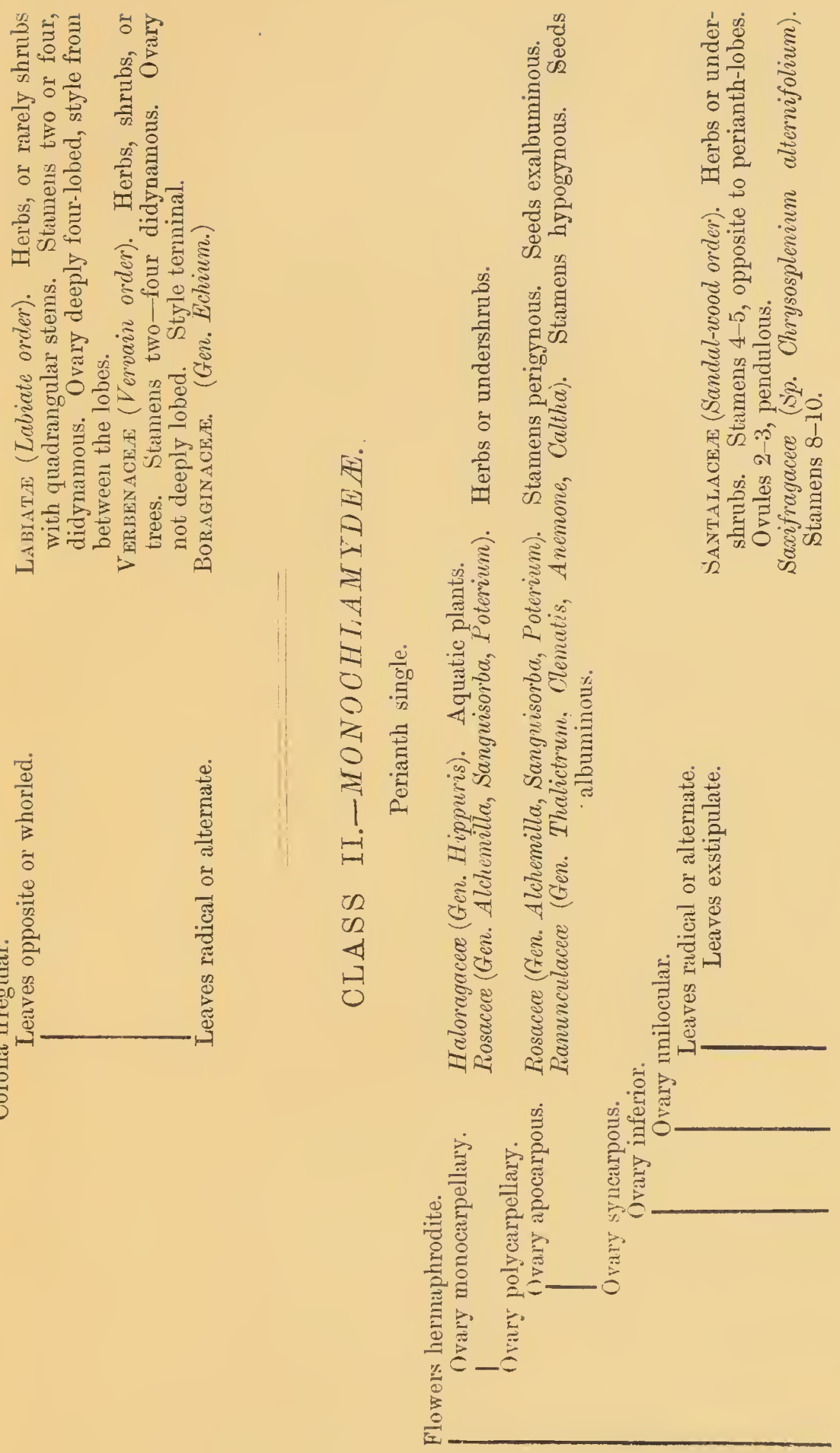


\section{(1!)}

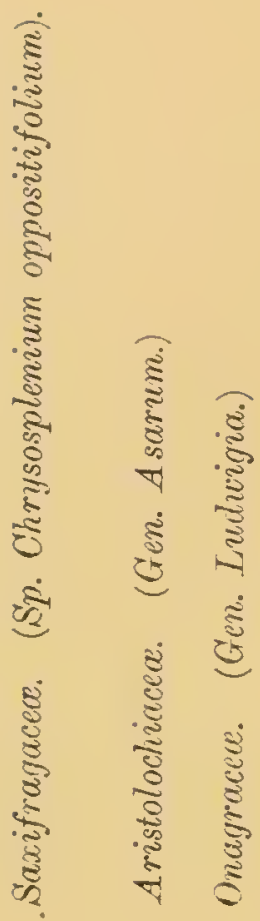

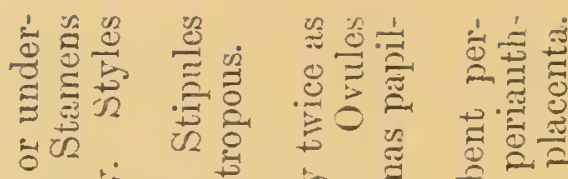

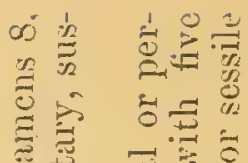

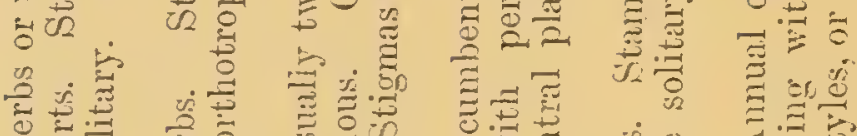

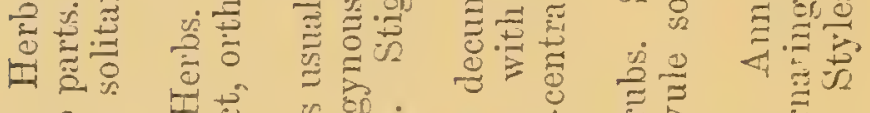

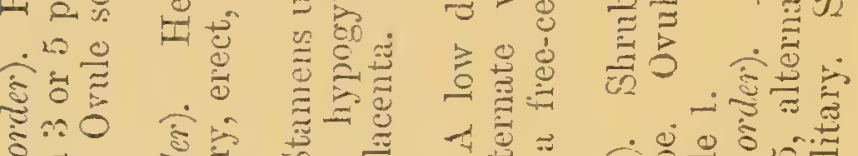
. .

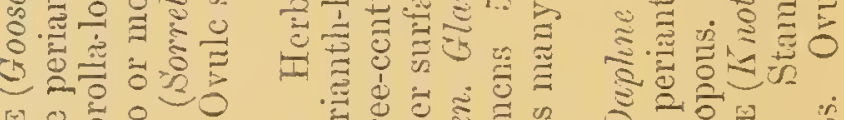

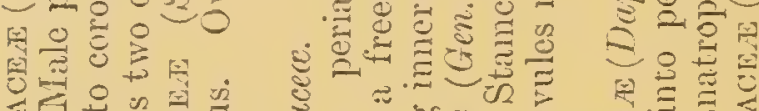

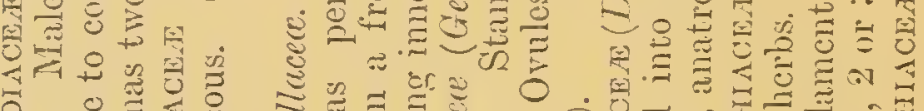

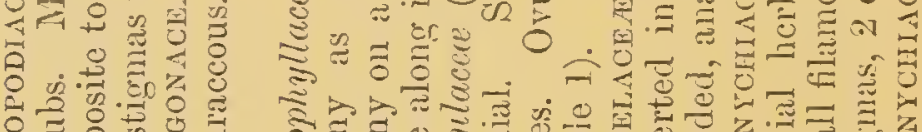

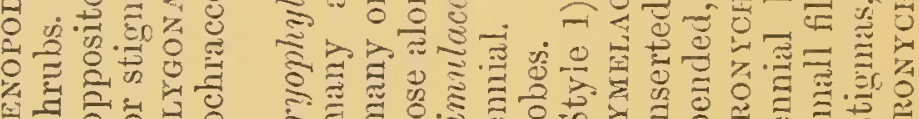

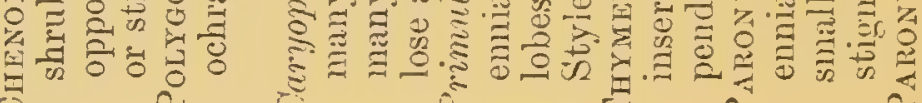

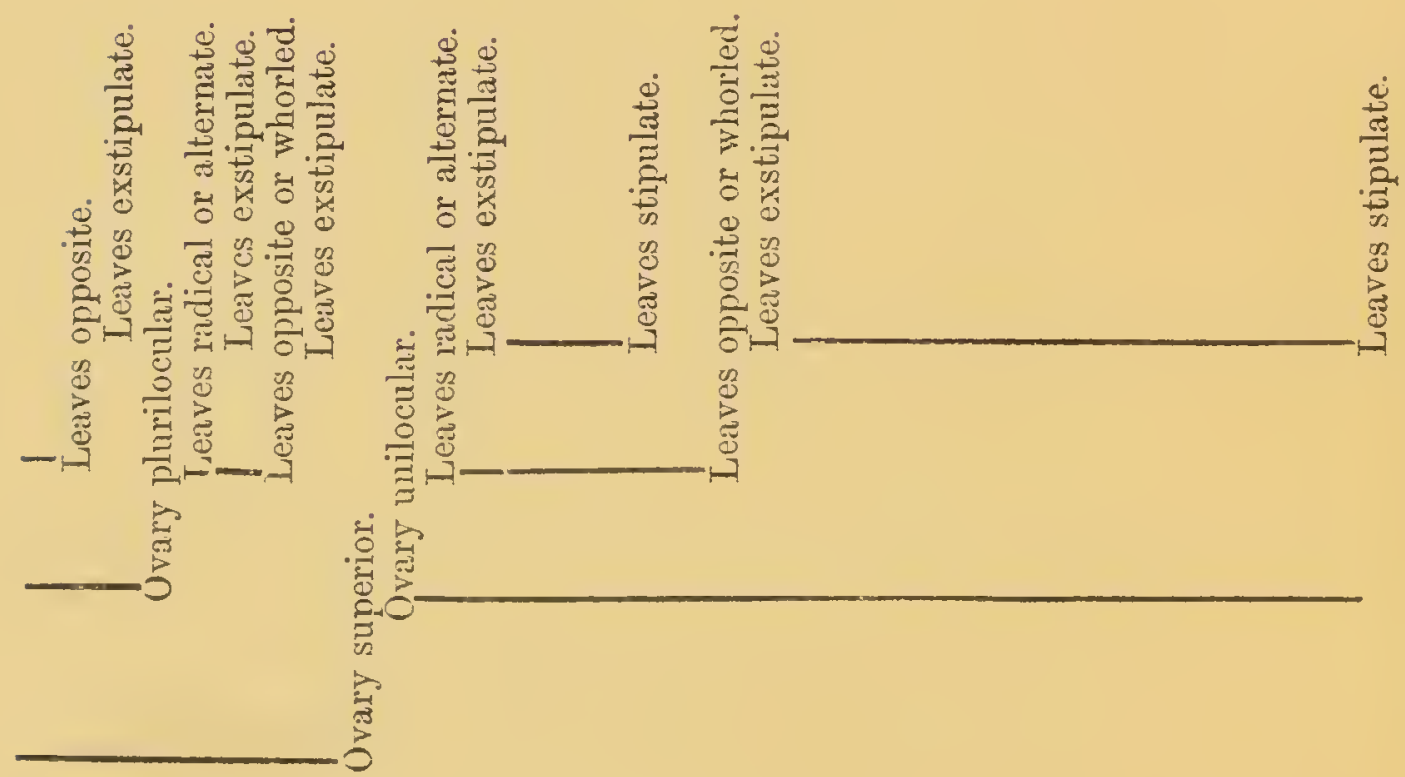


$(15)$
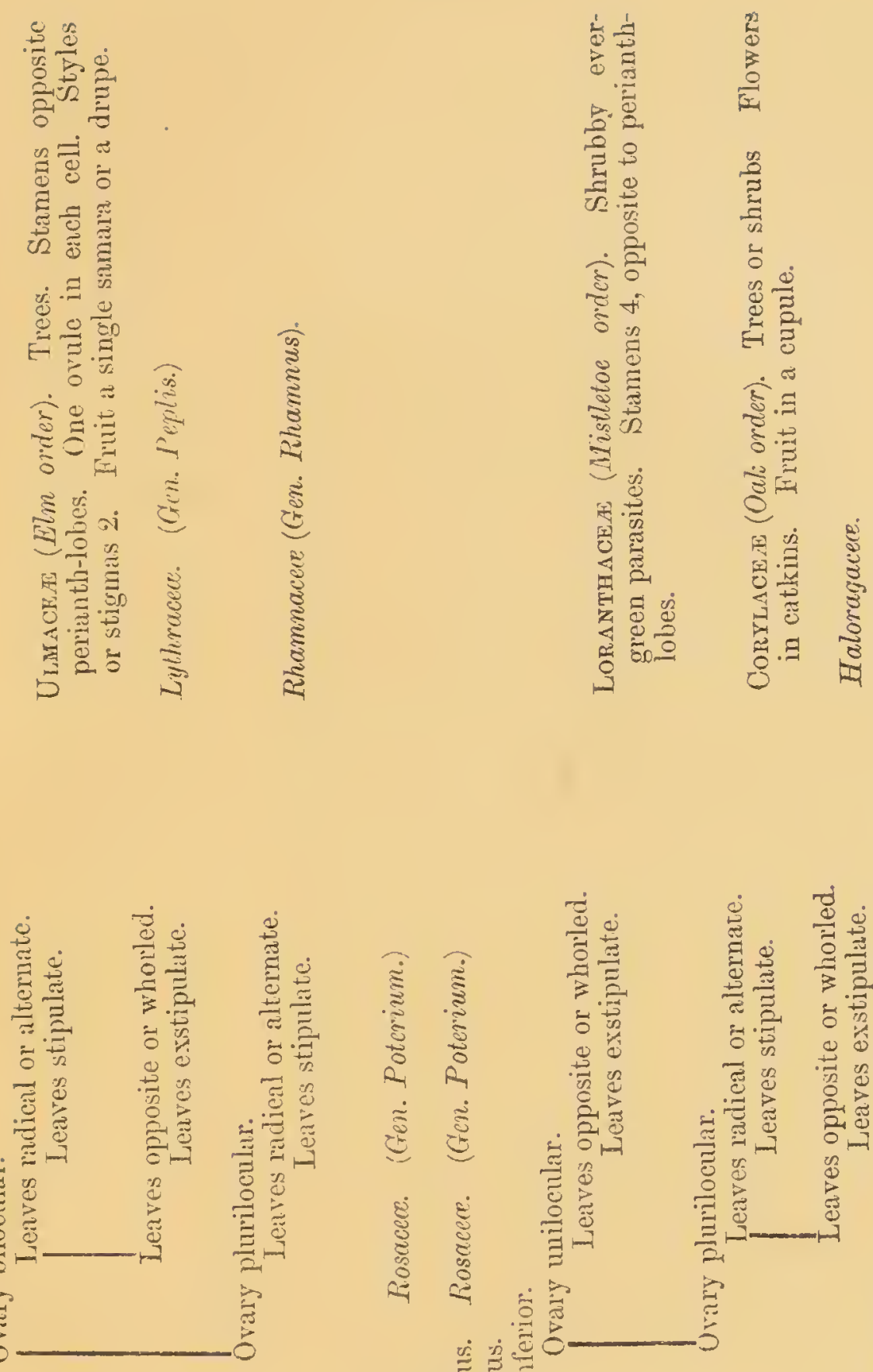

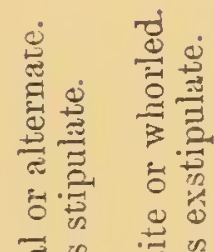

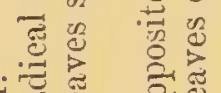

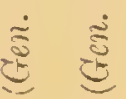

善告
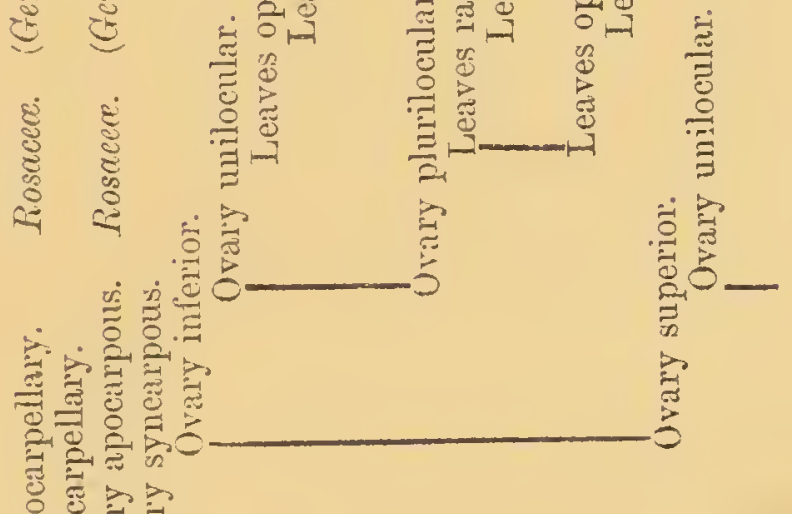

ค를

ڤڤ⿱宀㠯心㔾

政

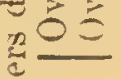




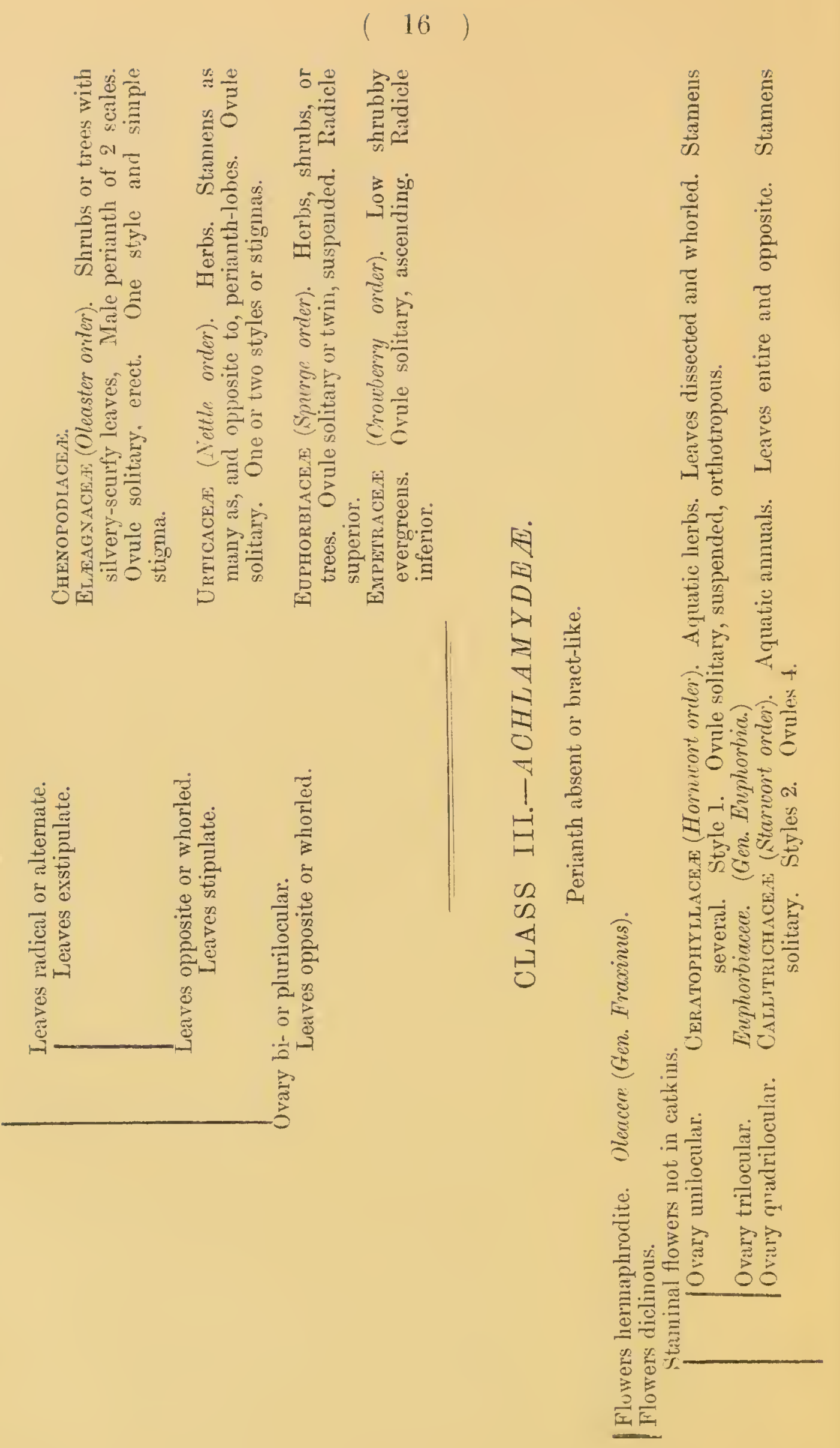


(17)
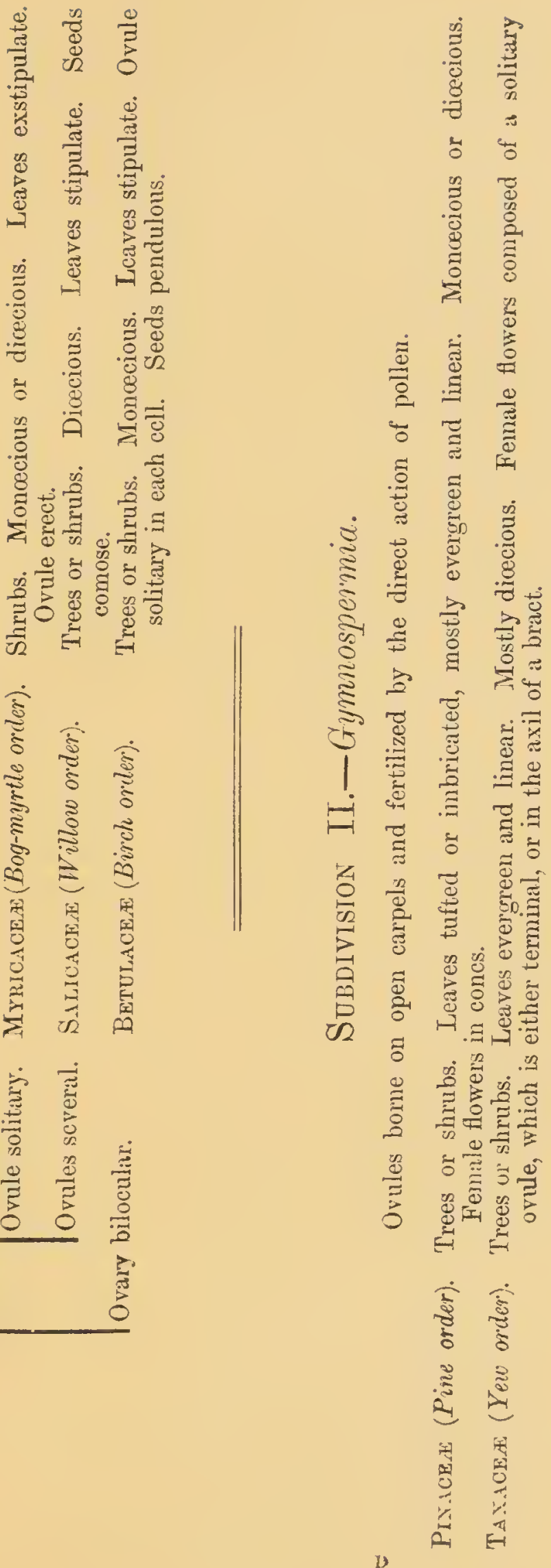


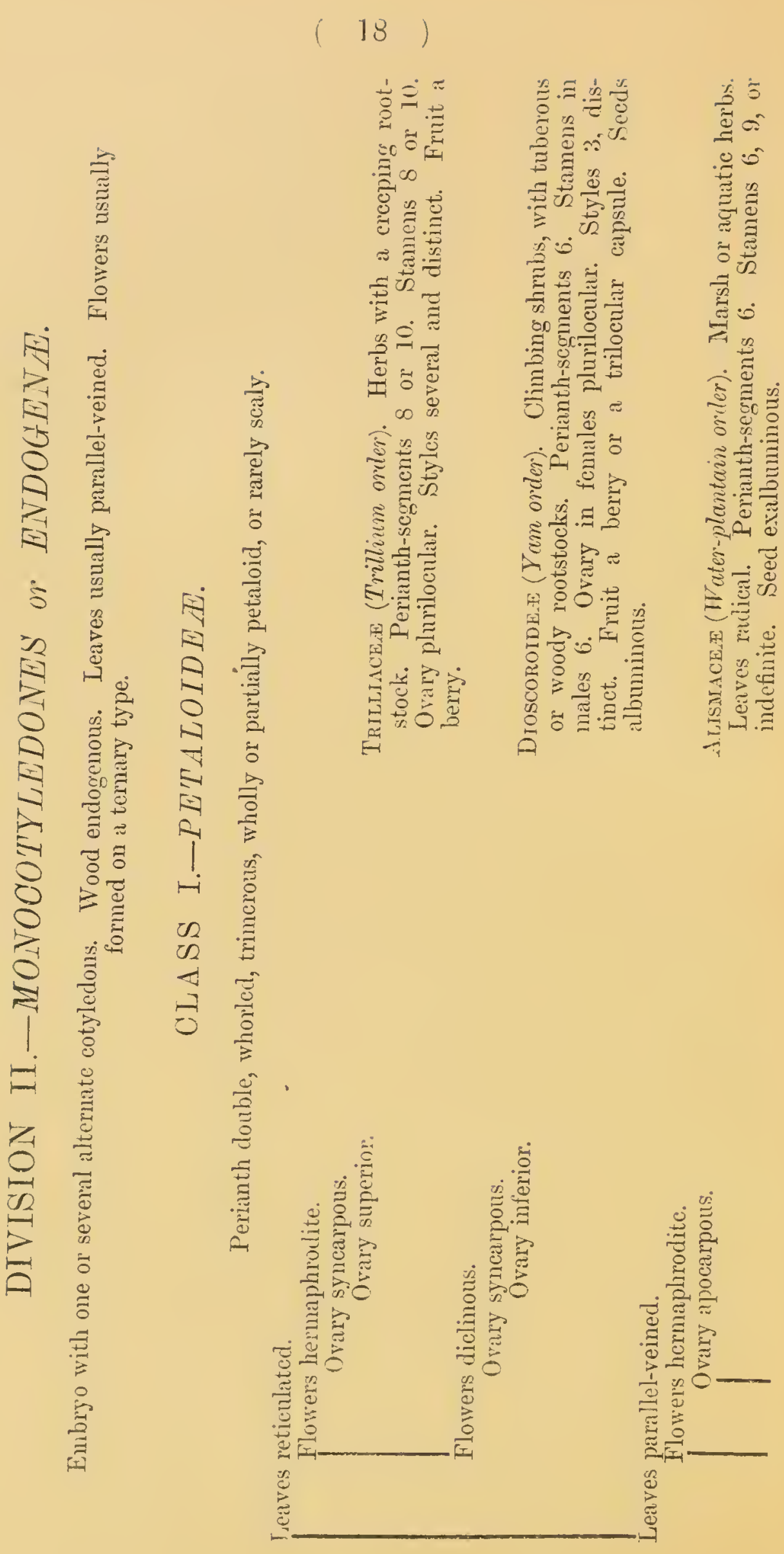




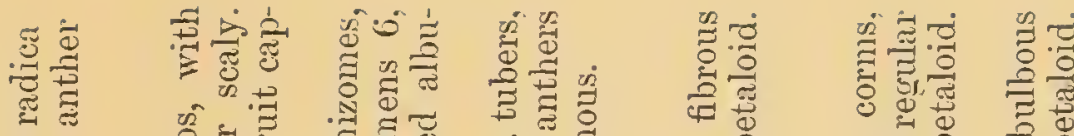
की

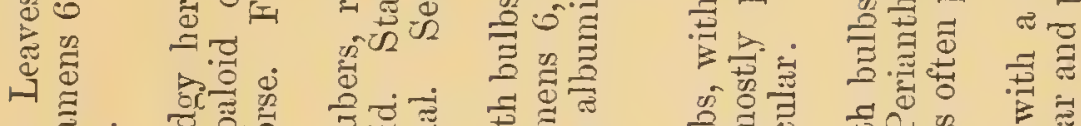

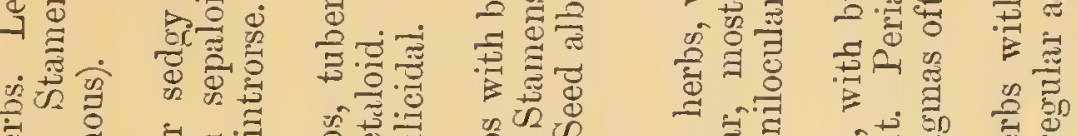

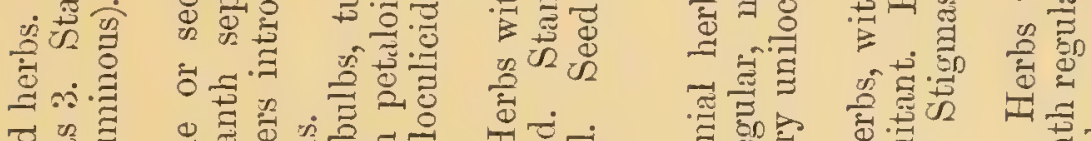

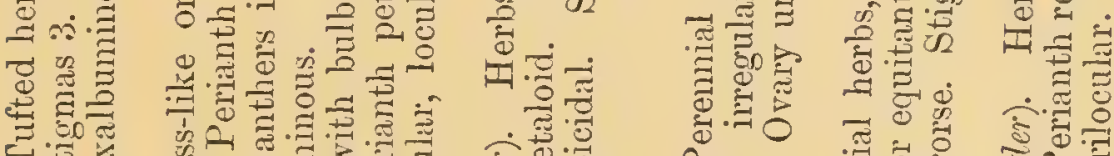

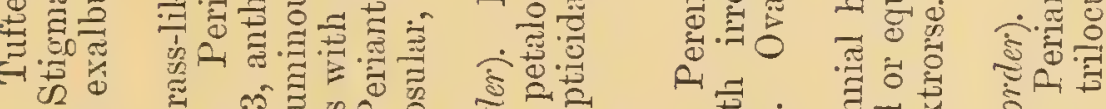

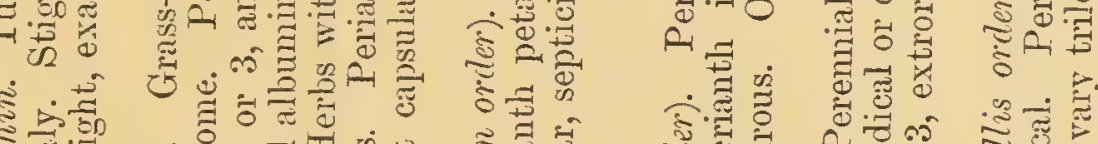

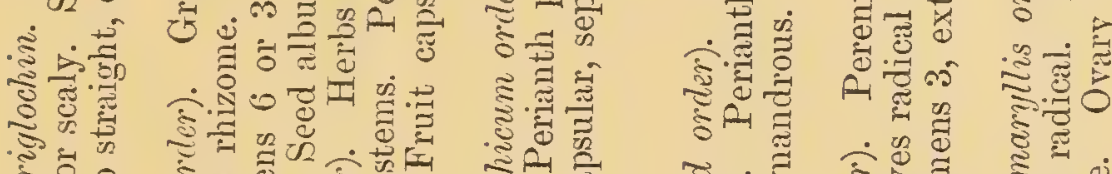

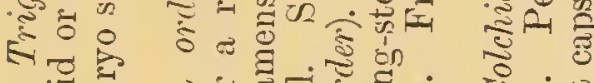

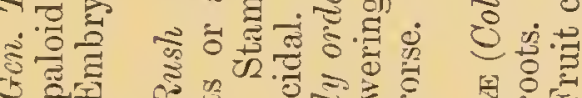

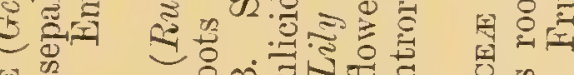

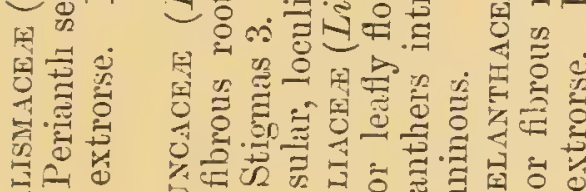

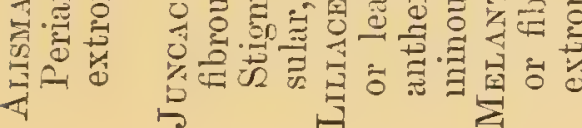

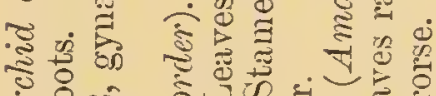
58 of के

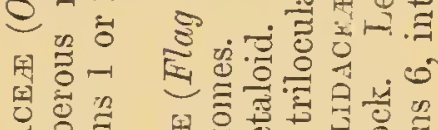

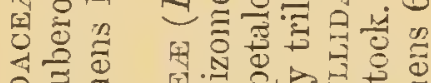

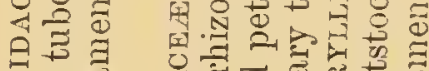

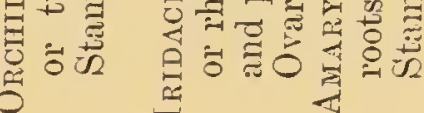

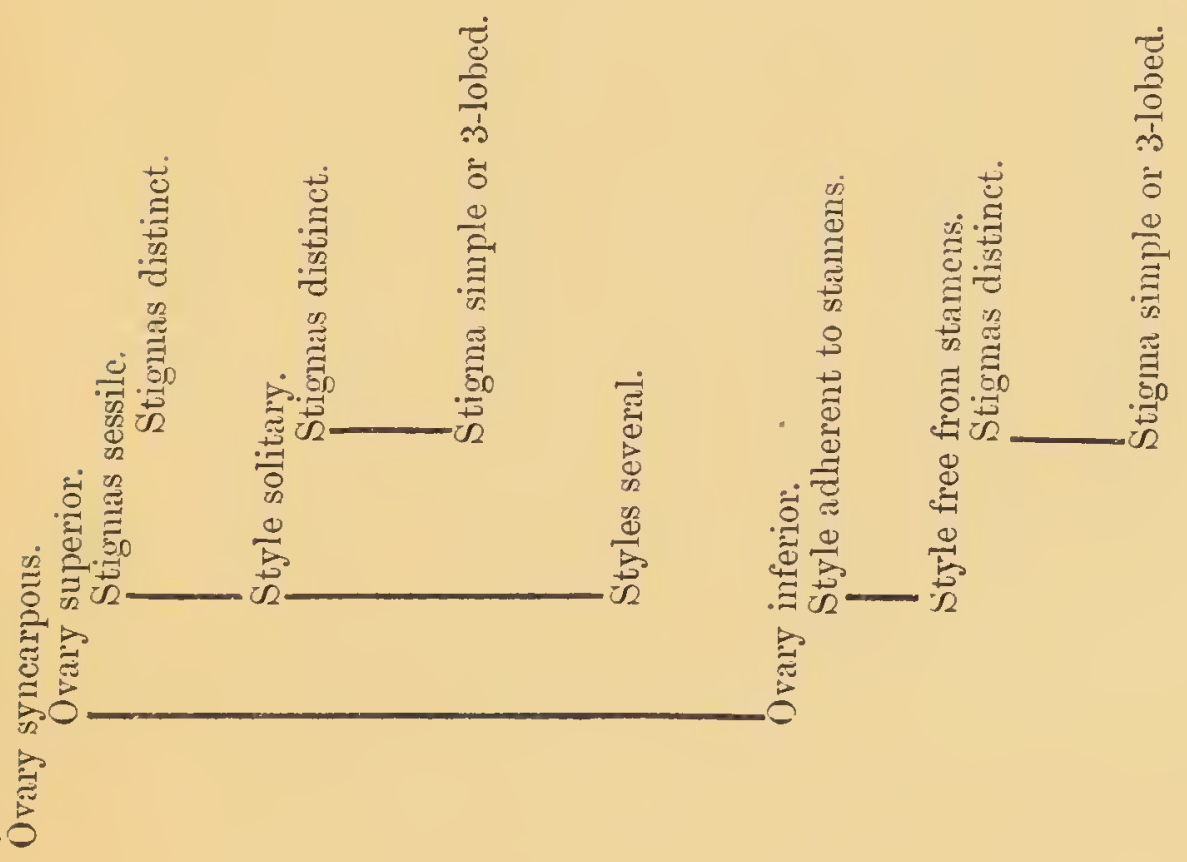


$(20)$
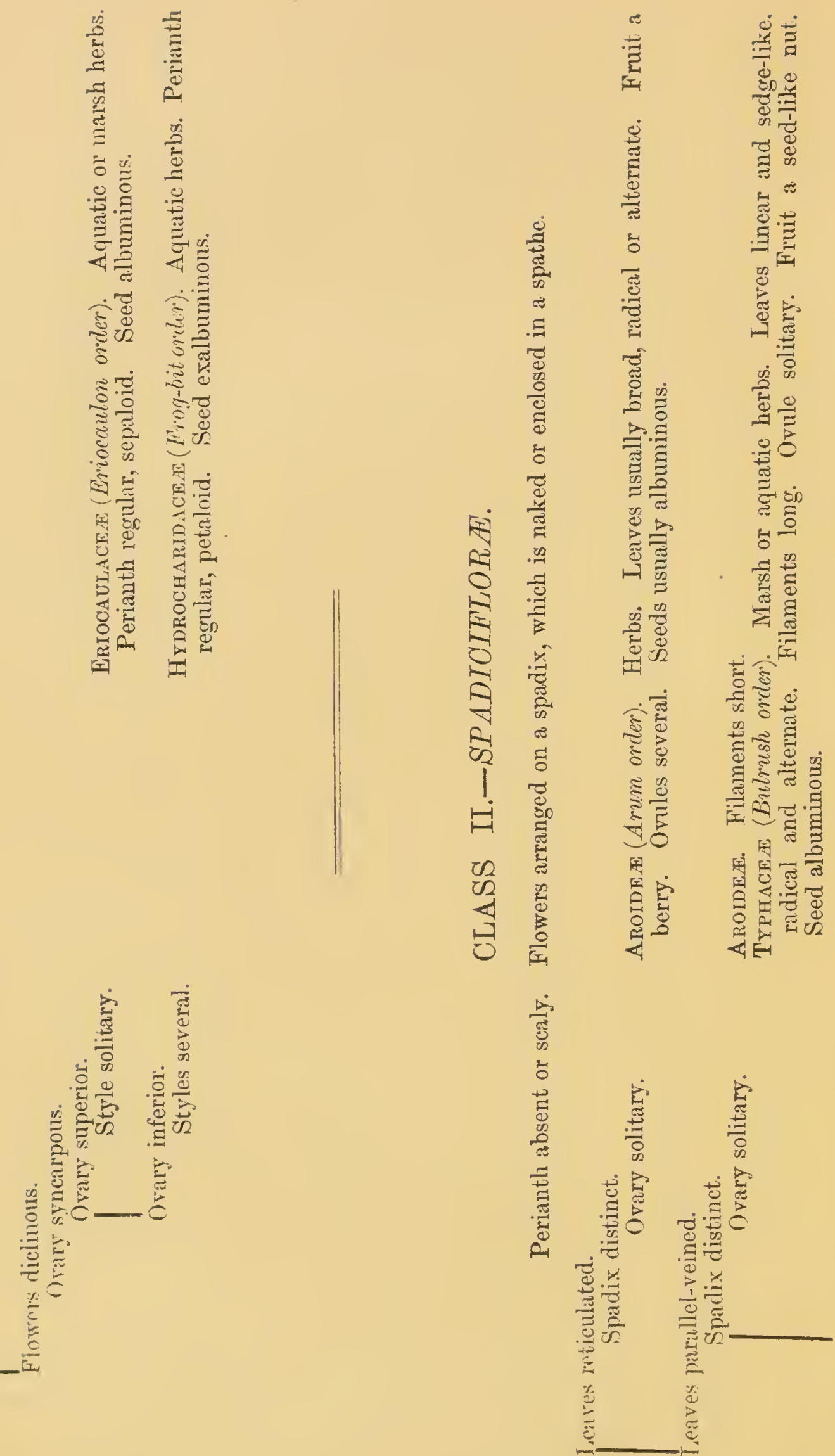


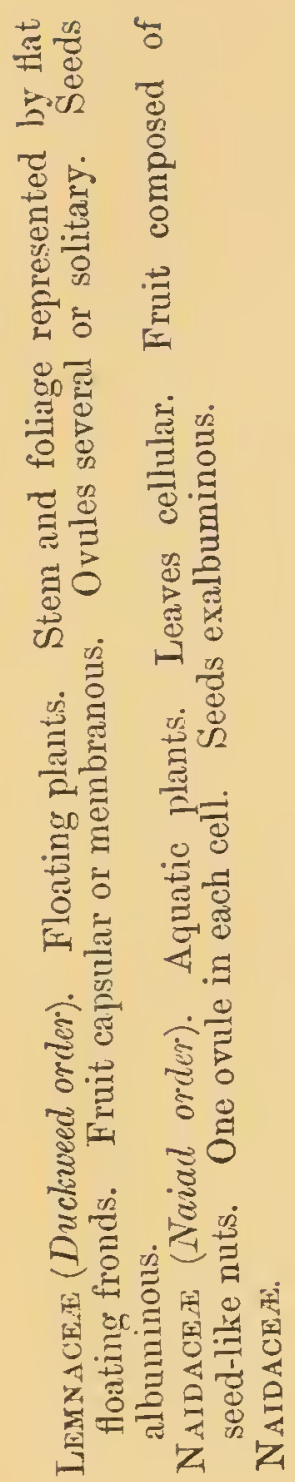

总离

$\left.\Xi \frac{0}{3}\right)$

㟧 온.콣 궁 둥응 룽. 苛臼䒕 눙 궁 . ज्ञ

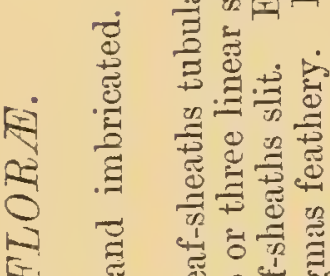

$\exists$ है

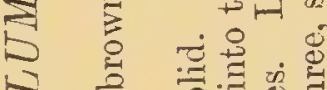

구

1 o

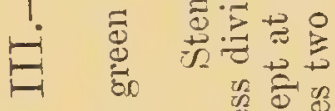

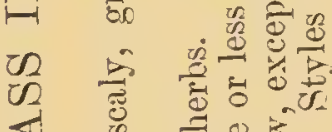

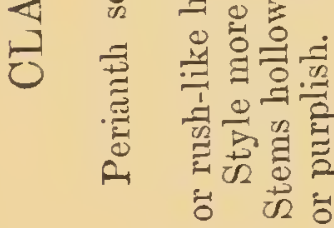

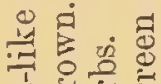

语

语究

ㄷํㄹ

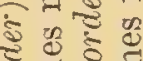

要专

콩

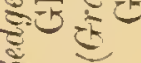

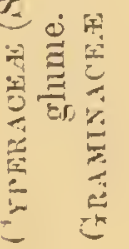


$2.2)$

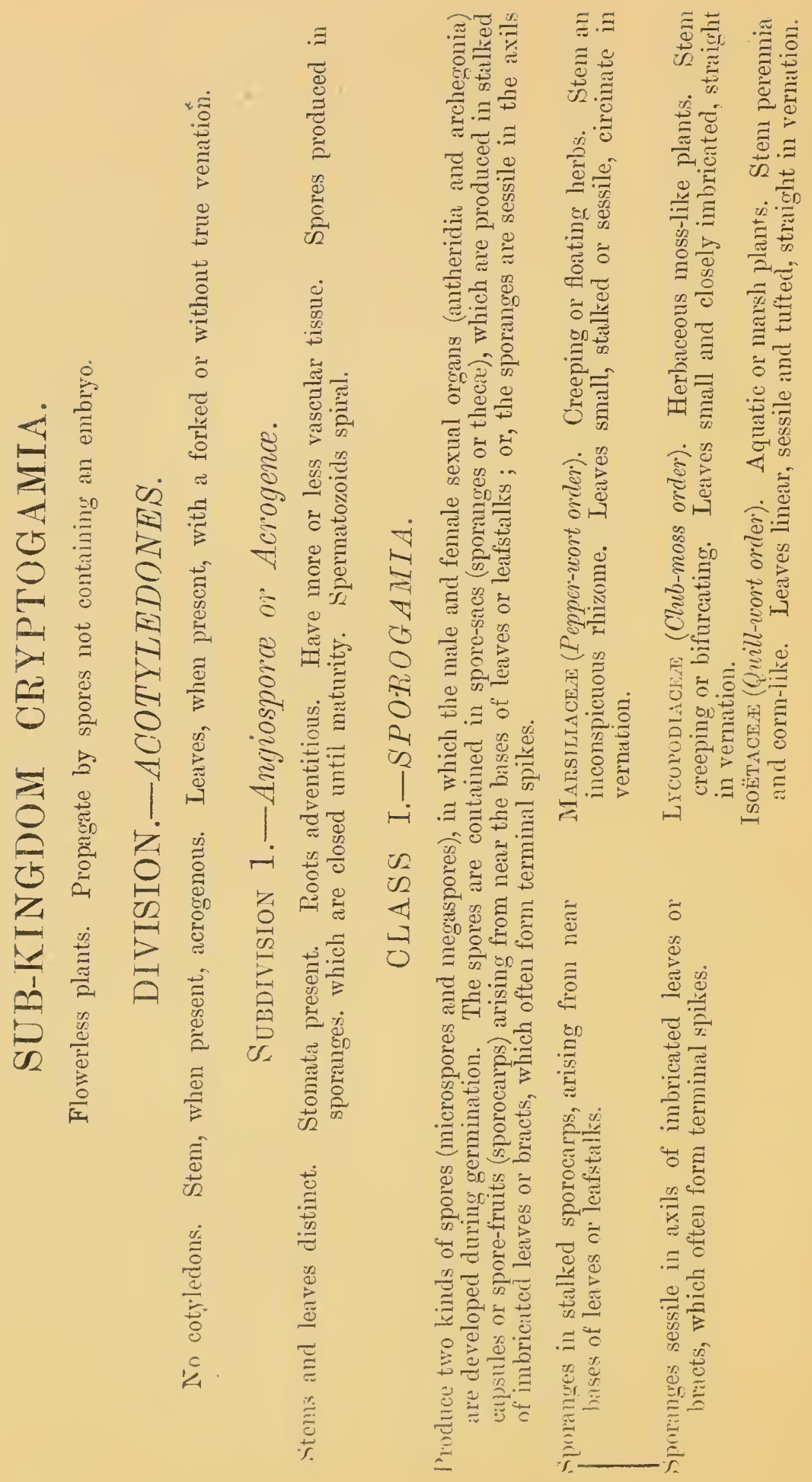




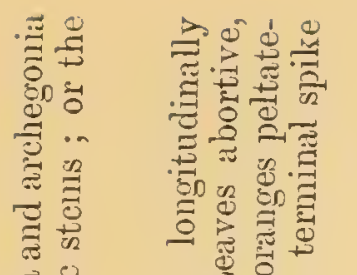

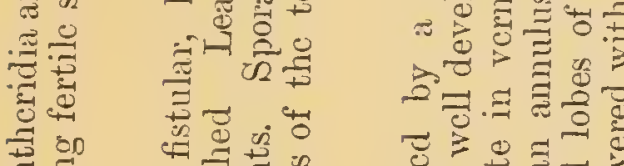

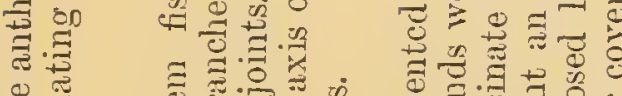

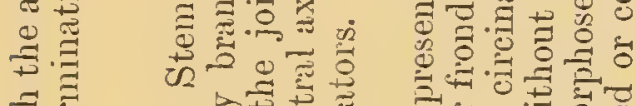

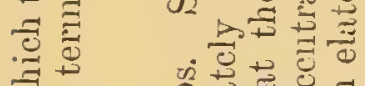

है. की

药

$\because \bar{\pi}$.

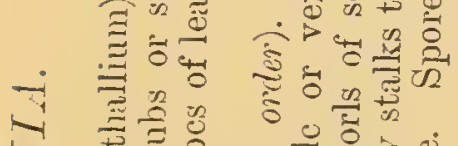

के

ヨ 0.0

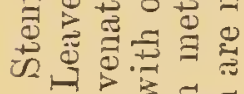

के

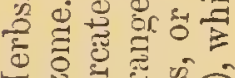

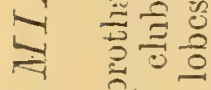

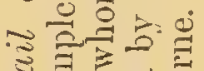

료밈

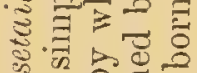

월

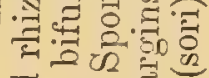

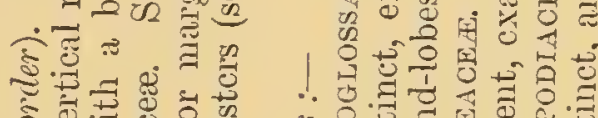

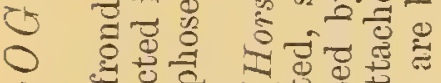

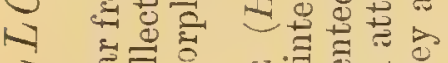

च

用.드웛

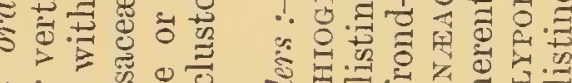

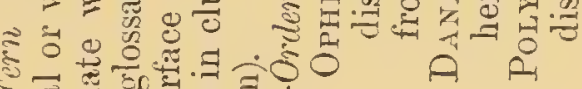
송율 굴 प्रें क्षें (

규유

- $\quad$ हc

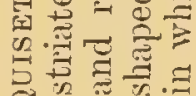

(t)

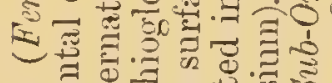

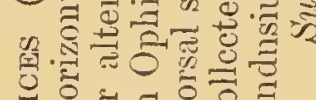

苟苍.

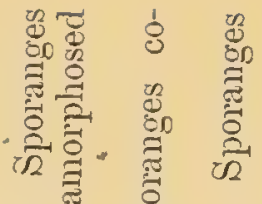

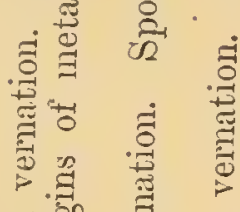

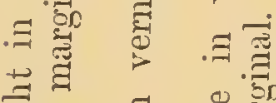

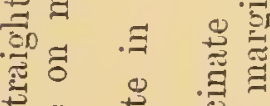

㻤

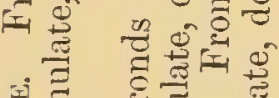

㱐急

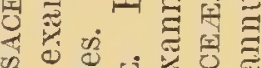
设

के की

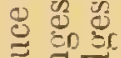

配

叫

ป

宊

范

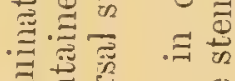

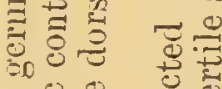

궁류

용

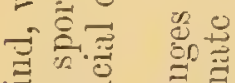

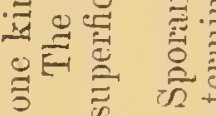

故葶

कै०े की

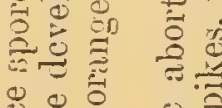

일잉

ع

สี ఫ్ర

芯言

$0_{0}=$

후

官.

ن

के

0 :

实

芯范

$=\frac{\pi}{\pi}$

응으 


\section{(24)}

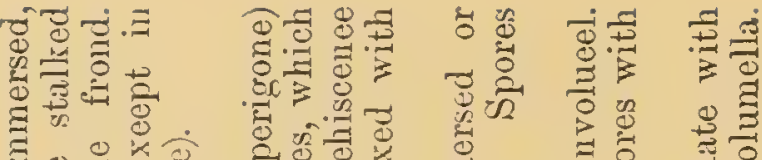

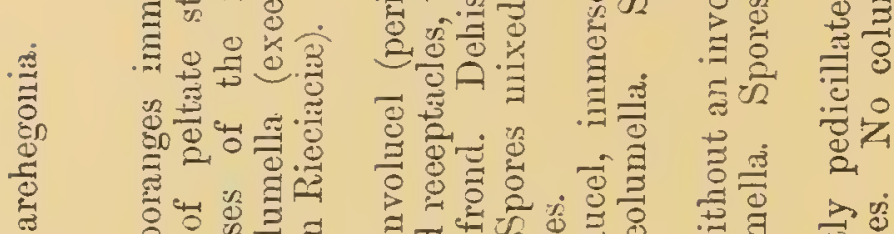

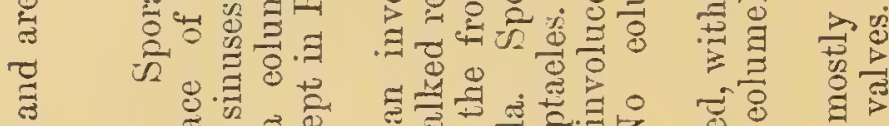

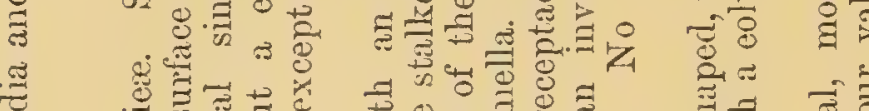

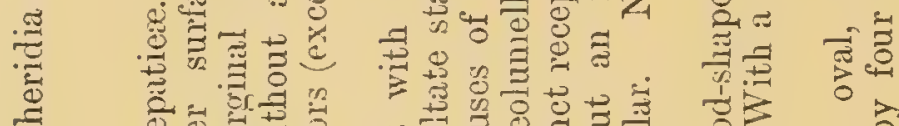

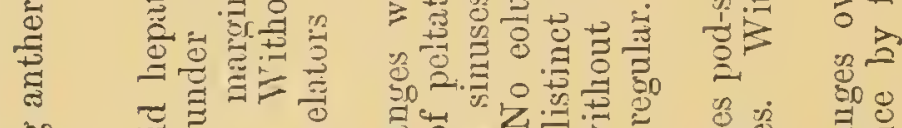

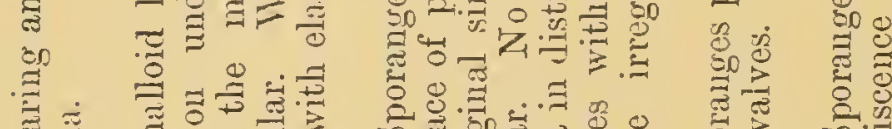

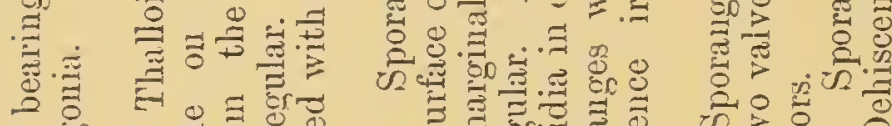

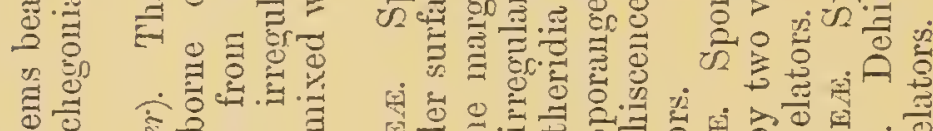

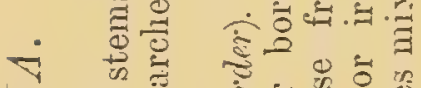

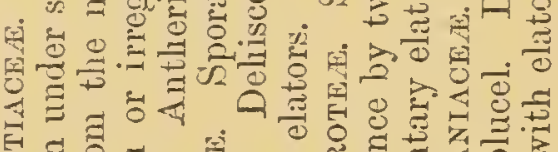

乙

४

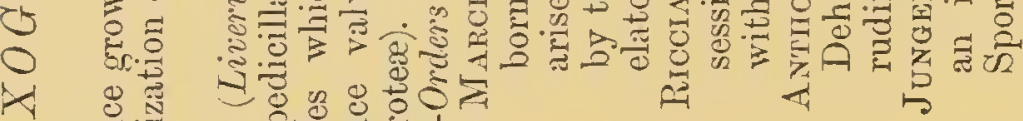

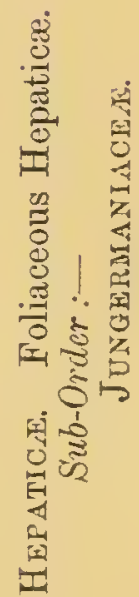

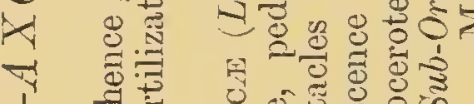

I

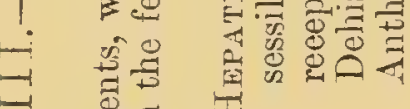

二

in

य

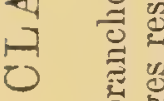

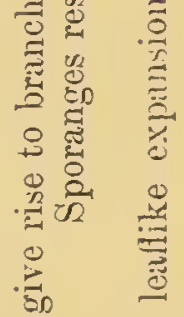

id

茟

ํㅕㄹ

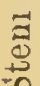

$\Xi$ 次

है

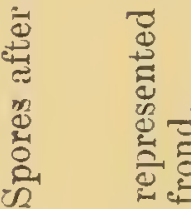

ब. 


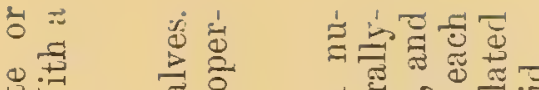

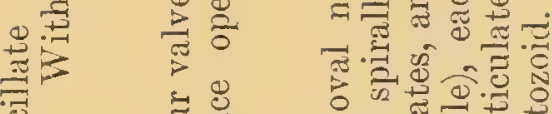

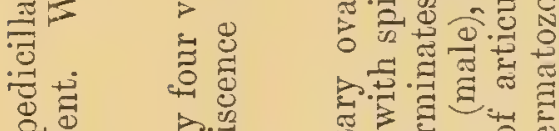

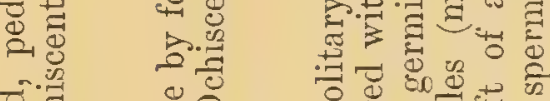

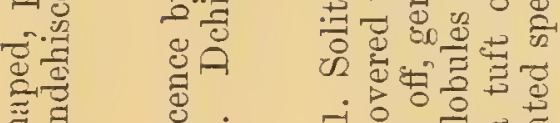

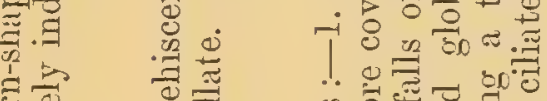

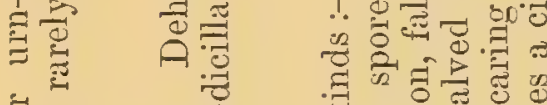

$\because 0$ 语

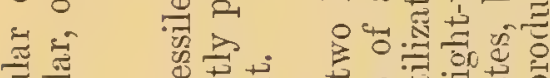

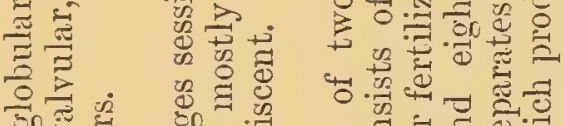

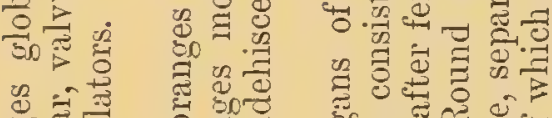

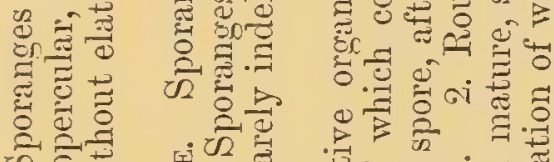

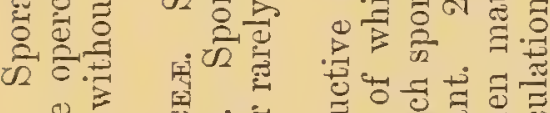

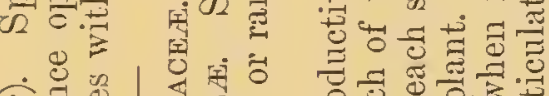

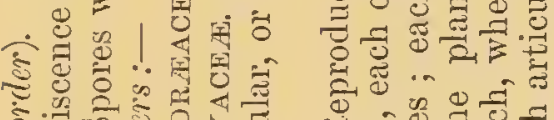

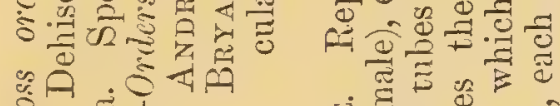

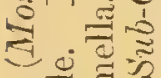

눌

岱
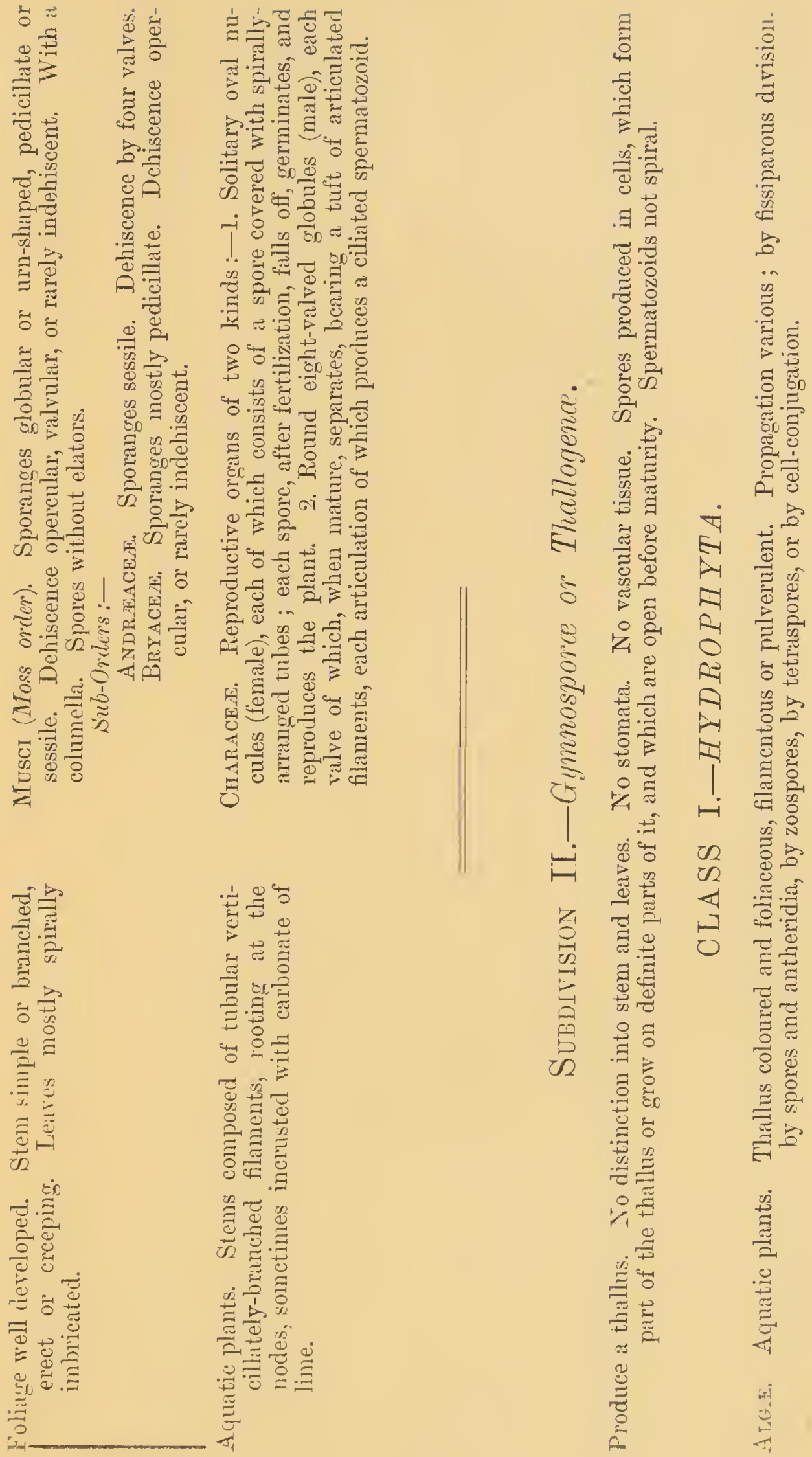

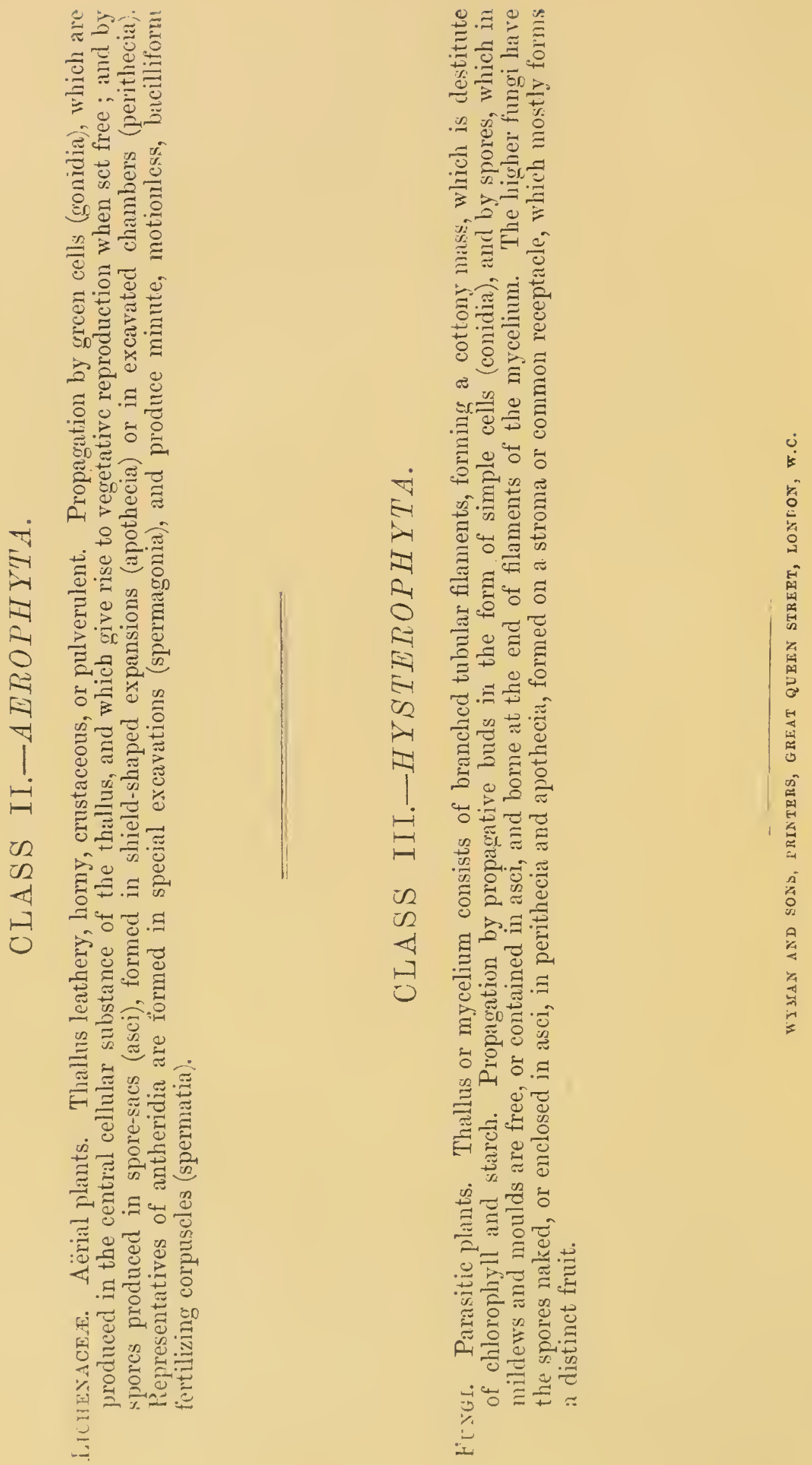


\section{NOTRS IND CORTRCTIONS.}

Fuge 2.- The Acted of Ranuncuraces: has a monocarpellary ovary.

'The leaves of FRANKENIACE are exstipulate.

I'urge 5.-Euntinacen.-For annual Mareh plants, read annual mersh plants.

P'rge (j.- The placentation of SAXIFHAGLEE is axile, not parietal.

The placentation of PARONYCHIACEA is not parietal, the ovule being solitary on $a$ basal funicle.

Puge 7.-In Rhamnaces and Celastraces, the ovules arise from the base of cells.

Page 8.-The petals of UyBELLIFEr are sometimes unequal.

Prege 9. - The ovary is sometimes bilocular in LobEtracezx.

„ Lenves are often alternate or scattered in Eircacex.

P'uge 10.- The ovary of VALERLAN.ICF, is more properly trilocular, two of the cells being abortive or empty.

Page 11.-The leaves of RuBIACE. have not invariably interpetiolar stipules.

I'uge 16. Leaves of Parictarin of URTrC.icr.s: are alternate and exstipulate.

, Lenves of HUPHORBICE. F and FuPETRACEA are not always opposite or whorled.

I'uge 19. - The perianth in Iris of TRIDAClats, aul in Calanthus of AMIRTLIIDACE.N, is irregular. 

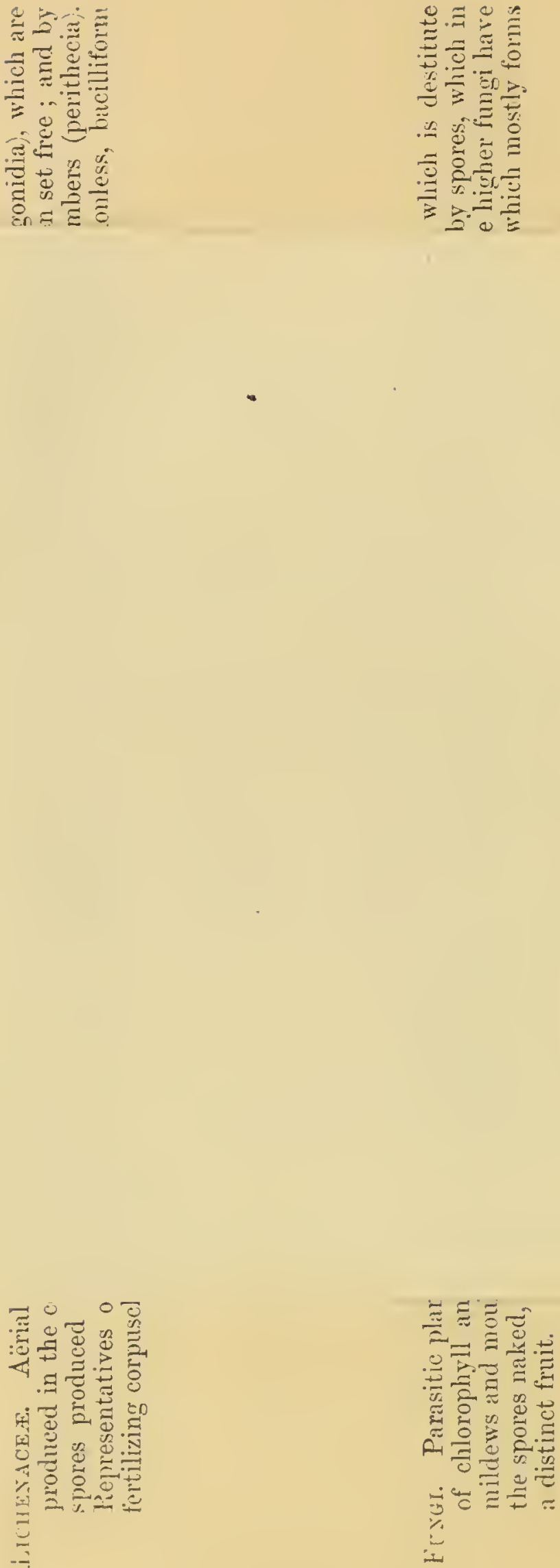





